\title{
6. NEOGENE RADIOLARIANS FROM THE EASTERN TROPICAL PACIFIC AND CARIBBEAN, DEEP SEA DRILLING PROJECT LEG 681
}

\author{
W. Riedel and M. Jean Westberg, Scripps Institution of Oceanography, La Jolla, California
}

\begin{abstract}
Two sites were drilled with the hydraulic piston corer on Leg 68 . Site 502, in the western Caribbean, contained radiolarians only in the uppermost (Quaternary) and lowermost (upper Miocene) cores. At Site 503, in the eastern Pacific, radiolarians were common and well preserved throughout the sequence, which terminated in the upper Miocene. The sequence of radiolarian events at Site 503 correlates well with that at Site 495, Leg 67, and the few discrepancies are resolved with an index of reliability. A detailed study of morphological changes during the evolution of Didymocyrtis spp. in the undisturbed sequence from Site 503 shows more departures from a simple rectilinear tendency than had been known from previous, coarser investigations.
\end{abstract}

\section{INTRODUCTION}

The recently developed hydraulic piston corer, with its capability of collecting relatively undisturbed samples, was used at the two sites occupied during this leg of the Deep Sea Drilling Project. At Site 502 in the western Caribbean $\left(11^{\circ} 29.5^{\prime} \mathrm{N}, 79^{\circ} 22.7^{\prime} \mathrm{W}\right.$; water depth 3052 $\mathrm{m})$, Quaternary radiolarians occured in the top two cores, but below that no siliceous microfossils were found until the lower upper Miocene was encountered near the bottom of the hole. This stratigraphic distribution of radiolarians is broadly consistent with that previously reported from the Caribbean (Riedel and Sanfilippo, 1973).

Radiolarians were common and well preserved throughout the sequence at Site $503\left(4^{\circ} 03.0^{\prime} \mathrm{N}, 95^{\circ} 38.2^{\prime} \mathrm{W}\right.$; water depth $3672 \mathrm{~m}$ ), which terminated in the upper Miocene. These cores are more useful for detailed radiolarian stratigraphy than those obtained earlier by rotary drilling; their only drawback is a reworked component estimated at about $1-70 \%$ that occurs in the top quarter of the column.

\section{PROCEDURES}

Two to three samples were taken generally, from each core, acidified to remove carbonate, sieved at $44 \mu \mathrm{m}$, and the coarse fraction strewn on slides according to conventional methods.

Relative abundances are recorded in two ways. First, in the middle of ranges and at most morphologic limits, five grades are used to indicate the abundance percentage of a taxon relative to the total assemblage. These percentage ranges are indicated, with their symbols, on Tables 1 and 2 .

Second, near evolutionary transitions and at morphotypic limits obscured by reworking, we used "constant-numerator" percentage estimates. Once the density of radiolarians is estimated, it is used to estimate the number of radiolarians in a counted number of fields that must be searched to find 10 specimens of a taxon (Riedel and Sanfilippo, 1978b).

In our investigation of radiolarians in the sequences cored on Leg 67 , we applied a newly developed procedure for evaluating the stratigraphic reliability of upper and lower limits of taxa and of evolutionary transitions (Westberg and Riedel, in press). Since this pro-

\footnotetext{
${ }^{1}$ Prell, W. L., Gardner, J. V., et al., Init. Repts. DSDP, 68: Washington (U.S. Govt. Printing Office).
}

cedure was also used for the radiolarian events at Site 503, we summarize the basis for calculating the index of reliability. The index (a number up to 100) is obtained by multiplying the following scores and expressing the resulting fraction as a percentage:

1) Abundance of the taxon -1 specimen observed in the sample scores $0.25 ; 2-5$ specimens, 0.50 ; more than 5 specimens, 1.00 .

2) For evolutionary transitions, the average of the relative abundances of ancestor and descendant through an appropriate distance above and below the event-if the average of descendant/ancestor counts above the event and ancestor/descendant counts below the event is $<2 x$, the score is $0.50 ; 2-5 x, 0.75,>5 x, 1.00$.

3) Type of event-a cryptogenetic origin or extinction scores 0.50 ; evolutionary extinctions and morphotypic limits bracketing an evolutionary transition, 0.75 ; an evolutionary transition, 1.00 .

4) Ease of distinguishing the taxon from co-occurring fossils-difficult, 0.10 ; moderately easy, 0.50 ; very easy, 1.00 .

5) Preservation of the assemblage relative to the taxon-very poor, 0.10 ; some specimens poor or uncertain, 0.50 ; good, 1.00 . This category is also used to downgrade the score of an upper limit above which (or lower limit below which) absence of the taxon is suspected to be due to dilution or dissolution.

6) Constancy of occurrence in the sequences above or below the event (not applied to evolutionary transitions)-interrupted, 0.75; constant, 1.00 .

7) Relation of locality to periphery of the area of distribution of the taxon-near periphery, 0.25 ; unknown, 0.50 ; well within, 1.00 .

8) Effects of reworking-investigator is confident that a range extended beyond his event determination is due to reworking, 0.75; lower scores are assigned in proportion to his uncertainty that the true range can be distinguished from reworking.

Having applied this procedure of evaluating the reliability of some 150 events from 7 sites drilled on Legs 67 and 68, it is appropriate to see whether the values of the indices are clustered in a certain part of the range from 1 to 100 or are more or less evenly spread. An even distribution would indicate that the index is suitably sensitive. Figure 1 shows the frequency of use of values of the reliability index, plotted on an antilog scale (because of the successive multiplications of fractions), for the upper Cenozoic sequences of Legs 67 and 68 and the lower to middle Miocene sections of Leg 67. The former show a satisfactory distribution of values throughout the range, but the indices of reliability for the older sequences in Leg 67 are clustered within the high end of the scale (above 25). The major reason for this concentration of values appears to be the high proportion of evolutionary transitions in early and middle Miocene radiolarian stratigraphy; other factors are the ease of recognition of taxa and the greater abundance, less reworking, and better preservation of the assemblages in the lower parts of the sequences of Leg 67.

Thus the values chosen for the multipliers used in calculating the reliability index have proven satisfactory in this application.

The radiolarian zones used in this chapter for the Pliocene and Miocene are the chronozones defined by Riedel and Sanfilippo (1978a). The Quaternary zones are those defined by Nigrini (1971). 


\section{W. RIEDEL, M. J. WESTBERG}

Table 1. Abundances of some radiolarians from Hole 503A.

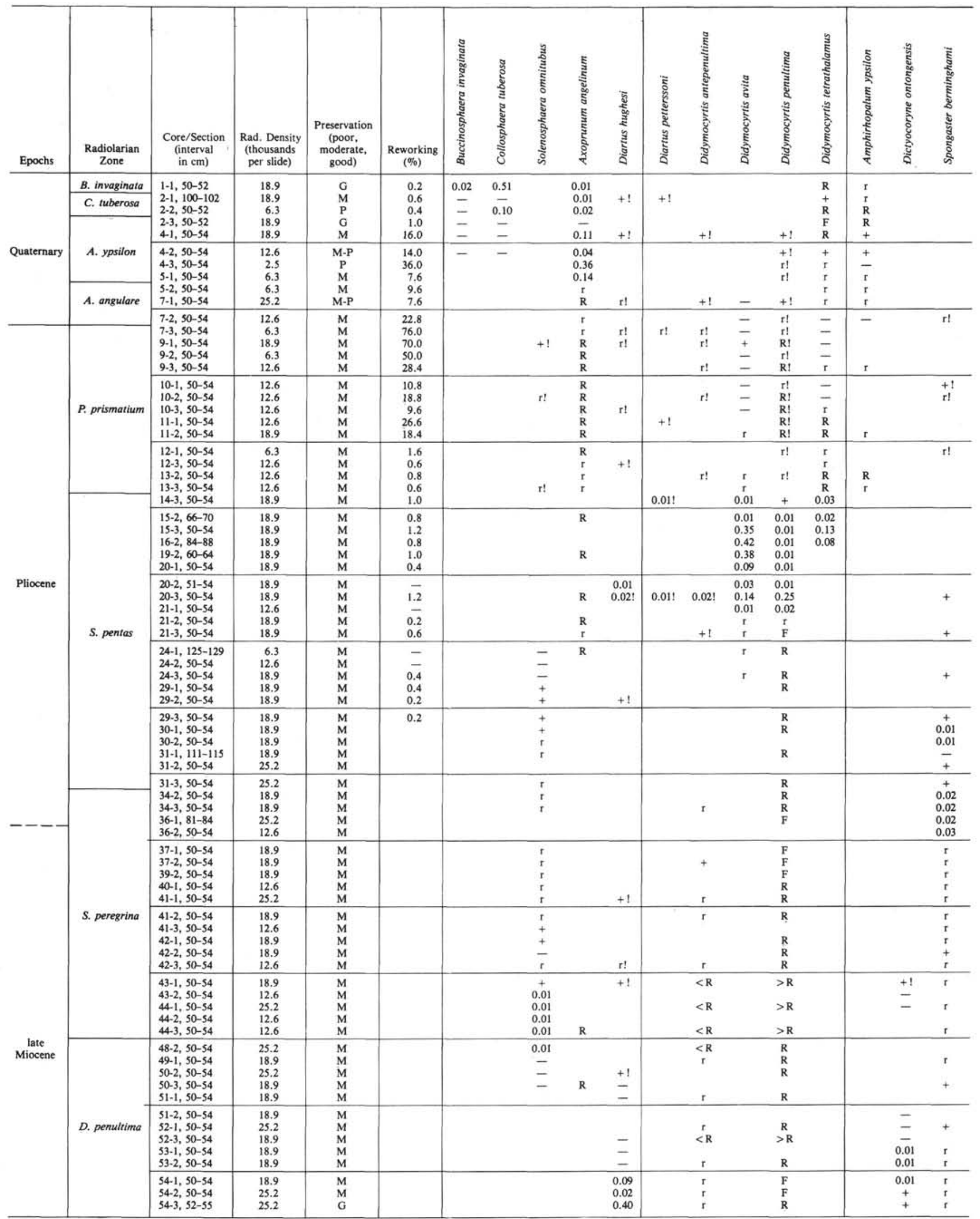

Note: $-=$ species sought but not found; $+=<0.01 \%$ of the radiolarians on the slide; $r=$ very rare $(0.01-0.1 \%) ; R=$ rare $(0.1-1 \%) ; F=$ few $(1-10 \%) ; !=$ reworking. $>$ and $<$ are used to indicate the dominant taxon when an ancestor and descendant abundance falls in the same percentage range. At critical points in ranges, numbers indicate abundance in percentage of total radiolarians on the slide as determined by constant-numerator counts (see text). 
Table 1. (Continued).

\begin{tabular}{|c|c|c|c|c|c|c|c|c|c|c|c|c|c|c|c|c|c|c|c|c|c|c|c|}
\hline $\begin{array}{l}\text { s. } \\
\text { है } \\
\text { हू } \\
\text { है } \\
\text { के }\end{array}$ & 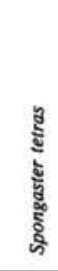 & 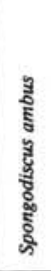 & 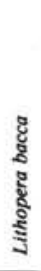 & 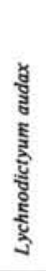 & $\begin{array}{l}\text { है } \\
\text { हू } \\
\text { हू } \\
\text { है } \\
\text { है } \\
\text { हूँ }\end{array}$ & 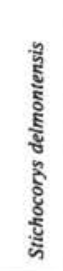 & 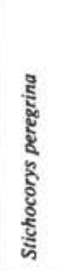 & 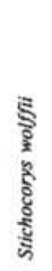 & 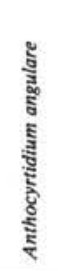 & 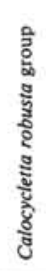 & 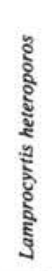 & 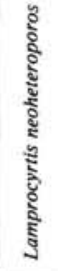 & $\begin{array}{l}\text { हू } \\
\text { है } \\
\text { है } \\
\text { है } \\
\text { है } \\
\text { है } \\
\text { है }\end{array}$ & $\begin{array}{l}\text { : } \\
\text { है } \\
\text { हू } \\
\text { है } \\
\text { हू } \\
\text { : }\end{array}$ & 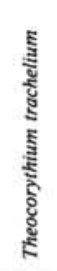 & 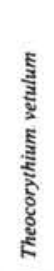 & 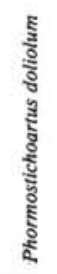 & 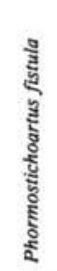 & 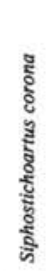 & 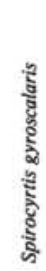 & $\begin{array}{l}\text { है } \\
\text { हूँ } \\
\text { है } \\
\text { है } \\
\text { है }\end{array}$ & $\begin{array}{l}\text { है } \\
\text { है } \\
\text { है } \\
\text { हैं } \\
\text { षे }\end{array}$ & 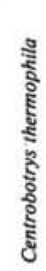 \\
\hline & $\begin{array}{l}\mathrm{r} \\
\mathrm{R} \\
\mathrm{R} \\
\mathrm{F} \\
\mathrm{R}\end{array}$ & 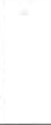 & $\begin{array}{l}\bar{z} \\
\bar{z} \\
\bar{r}\end{array}$ & & & $\begin{array}{l}+! \\
r ! \\
R ! \\
+1 \\
\mathbf{R} !\end{array}$ & $\begin{array}{l}+1 \\
r ! \\
r ! \\
\mathbf{R} !\end{array}$ & & $\begin{array}{l}\overline{-} \\
+! \\
=\end{array}$ & & & $\bar{z}$ & $\begin{array}{l}\mathrm{R} \\
\mathrm{r} \\
\mathrm{R} \\
\mathrm{R} \\
\mathrm{R}\end{array}$ & $\begin{array}{l}\mathrm{r} \\
\overline{+} \\
-\end{array}$ & $\begin{array}{l}- \\
\bar{r} \\
+ \\
R\end{array}$ & $\begin{array}{l}\bar{z} \\
\bar{r} !\end{array}$ & & & +1 & $\begin{array}{l}\bar{z} \\
\overline{+} \\
+\end{array}$ & $\begin{array}{l}\mathrm{r} \\
+ \\
\dot{\mathrm{R}} \\
-\end{array}$ & & $\begin{array}{l} \pm \\
\bar{z} \\
=\end{array}$ \\
\hline & $\begin{array}{l}\mathbf{R} \\
\mathbf{R} \\
\mathrm{r} \\
\mathrm{r} \\
\mathbf{R} \\
\end{array}$ & & $\begin{array}{l}\mathrm{r} \\
\mathrm{R} \\
\mathrm{r} \\
\mathrm{r}\end{array}$ & +1 & $\bar{z}$ & $\begin{array}{l}+1 \\
\mathrm{r} ! \\
\mathrm{r} ! \\
\mathrm{r} ! \\
\mathrm{r} !\end{array}$ & $\begin{array}{l}\mathrm{r} ! \\
\mathbf{R} ! \\
\mathrm{r} ! \\
\mathbf{R} ! \\
\mathbf{R} !\end{array}$ & & $\begin{array}{l}- \\
\bar{z} \\
\bar{R} \\
+\end{array}$ & $\stackrel{+!}{-}$ & $\begin{array}{l}\bar{z} \\
\bar{r} \\
0.01 \\
0.01\end{array}$ & $\begin{array}{l}\overline{-} \\
\overline{-} \\
0.05 \\
0.12\end{array}$ & $\begin{array}{l}\frac{\mathrm{r}}{\bar{T}} \\
0.02 \\
0.02 \\
0.01\end{array}$ & $\begin{array}{l}\bar{z} \\
\bar{r}\end{array}$ & $\begin{array}{c}\mathrm{r} \\
\mathrm{r} \\
0.27 \\
0.11 \\
0.12\end{array}$ & $\begin{array}{l}\bar{z} \\
\overline{1.27} \\
1.69\end{array}$ & $\begin{array}{l}+1 \\
- \\
+1\end{array}$ & & & $\begin{array}{l}\bar{r} \\
\bar{r} \\
+\end{array}$ & $\bar{z}$ & & $\bar{\Xi}$ \\
\hline & $\begin{array}{l}\mathbf{R} \\
\mathbf{R} \\
\mathbf{R} \\
\mathrm{r} \\
\mathrm{R} \\
\end{array}$ & & $\begin{array}{l}\mathrm{r} \\
\mathrm{r} \\
\mathrm{R} \\
\mathrm{r} \\
\mathrm{r}\end{array}$ & $\mathrm{r} !$ & $\begin{array}{l}\bar{r} \\
\mathrm{r} \\
\overline{-}\end{array}$ & $\begin{array}{c}\mathrm{R} ! \\
\mathrm{R} ! \\
\mathrm{r} ! \\
0.50 !\end{array}$ & $\begin{array}{c}\mathbf{R} ! \\
\mathrm{F} ! \\
\mathrm{F} ! \\
\mathrm{F} ! \\
0.89 !\end{array}$ & & $\begin{array}{l}\bar{z} \\
\bar{z}\end{array}$ & $\begin{array}{l}\mathrm{r} ! \\
\mathrm{r} ! \\
\mathrm{r} !\end{array}$ & $\begin{array}{c}0.32 \\
\overline{0.03} \\
R \\
R\end{array}$ & $\begin{array}{c}0.16 \\
\overline{0.01} \\
r \\
-\end{array}$ & $\begin{array}{l}\bar{z} \\
\bar{z}\end{array}$ & $\begin{array}{l}\mathrm{r} \\
=\end{array}$ & $\begin{array}{l}\frac{r}{z} \\
\end{array}$ & $\begin{array}{c}\mathrm{F} \\
\mathrm{F} \\
\mathrm{F} \\
\mathrm{F} \\
\mathrm{R}\end{array}$ & $\begin{array}{l}r ! \\
r ! \\
r ! \\
r !\end{array}$ & $\mathrm{r} !$ & & $\begin{array}{l}\mathrm{r} \\
\mathrm{r} \\
\mathrm{r} \\
\mathrm{r} \\
\mathrm{R}\end{array}$ & 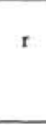 & +1 & $\mathrm{r}$ \\
\hline & $\begin{array}{l}\mathrm{R} \\
\mathrm{r} \\
\mathrm{R} \\
\mathrm{F} \\
\mathrm{R} \\
\end{array}$ & & $\begin{array}{l}t \\
R \\
R \\
t \\
\end{array}$ & $\frac{\mathrm{r} !}{+!}$ & $\begin{array}{l}- \\
\mathrm{r} \\
\mathrm{r} \\
+ \\
\end{array}$ & $\begin{array}{l}0.10 ! \\
0.32 ! \\
0.02 ! \\
0.35 ! \\
0.31 !\end{array}$ & $\begin{array}{l}0.38 ! \\
0.53 ! \\
0.551 ! \\
0.57 ! \\
0.51 !\end{array}$ & & & $\mathrm{r} !$ & $\begin{array}{l}\mathrm{R} \\
\mathrm{r} \\
\mathrm{R} \\
\mathrm{R} \\
\mathrm{R} \\
\end{array}$ & $\frac{r}{r}$ & & & & $\begin{array}{l}\mathrm{F} \\
\mathrm{R} \\
\mathrm{R} \\
\mathrm{F} \\
\mathrm{F} \\
\end{array}$ & $\begin{array}{l}\mathrm{r} ! \\
\mathrm{r} ! \\
\mathrm{r} ! \\
\mathrm{r} ! \\
\mathrm{r} !\end{array}$ & $\overline{\bar{r} !}$ & $\begin{array}{l}\mathrm{r} ! \\
\mathrm{r} !\end{array}$ & $\begin{array}{l}R \\
r \\
r\end{array}$ & & & $\begin{array}{l}r \\
r \\
r\end{array}$ \\
\hline & $\begin{array}{l}\mathbf{R} \\
\mathrm{R} \\
\mathrm{R} \\
\mathrm{R} \\
\mathrm{R} \\
\end{array}$ & & $\begin{array}{l}\mathrm{r} \\
\mathbf{R} \\
\mathbf{R} \\
\mathrm{R} \\
- \\
\end{array}$ & - & $\begin{array}{l}\bar{r} \\
\mathrm{r} \\
\mathrm{r} \\
\mathrm{r} \\
\end{array}$ & $\begin{array}{l}\overline{-.01 !} \\
0.01 ! \\
0.01 ! \\
0.04 !\end{array}$ & $\begin{array}{l}0.03 ! \\
0.08 ! \\
0.02 ! \\
0.04 ! \\
6.09\end{array}$ & & & $\mathrm{rl}$ & $\begin{array}{l}R \\
r \\
r \\
r \\
R\end{array}$ & $\begin{array}{l}\mathrm{r} \\
+ \\
\mathrm{r} \\
\mathrm{r}\end{array}$ & & & & $\begin{array}{l}\mathrm{R} \\
\mathrm{F} \\
\mathrm{R} \\
\mathrm{R} \\
\mathrm{R} \\
\end{array}$ & $\begin{array}{l}0.02 \\
0.01 \\
0.01 \\
-\overline{0} \\
0.01\end{array}$ & $\begin{array}{l}0.02 \\
0.01 \\
0.01 \\
0.01 \\
-\end{array}$ & & $\begin{array}{l}r \\
R \\
R \\
R \\
R\end{array}$ & & & $\begin{array}{l}r \\
r \\
R \\
R \\
R \\
\end{array}$ \\
\hline $\begin{array}{l}0.01 \\
\overline{0.01}\end{array}$ & $\begin{array}{c}\mathrm{F} \\
\mathrm{F} \\
\mathrm{F} \\
\mathrm{F} \\
0.11 \\
\end{array}$ & - & $\begin{array}{l}\bar{r} \\
\mathrm{r} \\
\mathrm{R} \\
\mathrm{r}\end{array}$ & $\begin{array}{l}\bar{z} \\
\overline{+} \\
+\end{array}$ & $\begin{array}{l}r \\
R \\
+ \\
r \\
r \\
\end{array}$ & $\begin{array}{c}0.03 ! \\
0.05 ! \\
0.04 ! \\
r !\end{array}$ & $\begin{array}{c}5.10 \\
9.00 \\
9.90 \\
F \\
F \\
\end{array}$ & & & $\begin{array}{l}+1 \\
+1 \\
\end{array}$ & $\begin{array}{l}\frac{r}{-} \\
-\end{array}$ & $\begin{array}{l}+ \\
+ \\
+\end{array}$ & + & & & $\begin{array}{l}R \\
R \\
R \\
R \\
R \\
\end{array}$ & $\begin{array}{l}\overline{0.01} \\
\overline{0.84} \\
0.06\end{array}$ & $\begin{array}{l}\overline{0.01} \\
0.01 \\
0.02 \\
0.02\end{array}$ & & $\begin{array}{l}\mathbf{R} \\
\mathbf{R} \\
\mathbf{R} \\
\mathbf{R} \\
\mathbf{R} \\
\end{array}$ & $\begin{array}{l}R \\
r \\
\end{array}$ & & $\begin{array}{l}R \\
r \\
r \\
R \\
R \\
\end{array}$ \\
\hline $\begin{array}{c}0.01 \\
0.04 \\
0.05 \\
r \\
r \\
\end{array}$ & $\begin{array}{c}0.01 \\
0.02 \\
= \\
=\end{array}$ & $\begin{array}{l}\overline{+1} \\
\overline{+} \\
+\end{array}$ & $\begin{array}{l}R \\
R \\
R \\
R \\
\end{array}$ & $\begin{array}{l}- \\
+ \\
+ \\
+ \\
+\end{array}$ & $\begin{array}{l}\mathrm{r} \\
\mathrm{r} \\
\mathrm{r} \\
\mathrm{r}\end{array}$ & $\begin{array}{l}\mathrm{r} ! \\
\mathrm{r} !\end{array}$ & $\begin{array}{l}\mathrm{F} \\
\mathrm{F} \\
\mathrm{F} \\
\mathrm{F} \\
\mathrm{F}\end{array}$ & & & $\begin{array}{l}\mathrm{r} ! \\
\mathrm{r} ! \\
\mathrm{r} !\end{array}$ & - & - & & & & $\begin{array}{l}\mathrm{R} \\
\mathrm{R} \\
\mathbf{r} \\
\mathrm{R}\end{array}$ & $\begin{array}{c}0.40 \\
0.83 \\
0.69 \\
R \\
R \\
\end{array}$ & $\begin{array}{c}0.20 \\
+ \\
+ \\
+\end{array}$ & $\mathrm{r} !$ & $\begin{array}{l}\mathbf{R} \\
\mathrm{R} \\
\mathrm{R} \\
\mathrm{R}\end{array}$ & ${ }_{\mathrm{r}}^{\mathrm{r}}$ & & R \\
\hline $\mathrm{r}$ & & $\begin{array}{l}t \\
+ \\
+ \\
+\end{array}$ & $\begin{array}{l}\mathrm{t} \\
\mathrm{r} \\
\mathrm{R}\end{array}$ & $\begin{array}{l}r \\
r\end{array}$ & $\begin{array}{l}\mathrm{r} \\
\mathrm{r} \\
\mathrm{r} \\
- \\
-\end{array}$ & $\mathrm{r} !$ & $\begin{array}{l}F \\
F\end{array}$ & +1 & & $\begin{array}{l}- \\
\overline{+} \\
-\end{array}$ & & & & & & $\begin{array}{l}+ \\
\mathrm{r} \\
\mathrm{r} \\
\overline{+}\end{array}$ & $\begin{array}{l}\mathrm{R} \\
\mathrm{F} \\
\mathrm{F}\end{array}$ & $\begin{array}{l}r \\
r \\
r\end{array}$ & $\begin{array}{l}\overline{+1} \\
\overline{-}\end{array}$ & $\mathbf{R}$ & $\begin{array}{l}\mathrm{r} \\
\mathrm{r} \\
\mathrm{r}\end{array}$ & & $\begin{array}{l}R \\
R \\
R\end{array}$ \\
\hline $\begin{array}{c}{ }^{r} \\
0.02 \\
0.01 \\
0.01 \\
0.01\end{array}$ & $\begin{array}{l}+ \\
\pm \\
+ \\
+\end{array}$ & $\begin{array}{l}\mathrm{r} \\
\mathrm{r} \\
\mathrm{r} \\
\mathrm{r}\end{array}$ & $\begin{array}{l}\mathbf{R} \\
\mathrm{R}\end{array}$ & $\begin{array}{l}+ \\
+ \\
+ \\
+\end{array}$ & $\overline{-}$ & $\mathrm{r} !$ & $\begin{array}{l}\mathrm{F} \\
\mathrm{F}\end{array}$ & & & $\begin{array}{l}+1 \\
= \\
=\end{array}$ & & & & & & $\begin{array}{l}\bar{z} \\
\bar{z}\end{array}$ & $\begin{array}{l}\mathrm{F} \\
\mathrm{R}\end{array}$ & $\begin{array}{l}r \\
+ \\
+\end{array}$ & $\begin{array}{l}\bar{t} \\
\mathbf{r} \\
\overline{-}\end{array}$ & $\mathbf{R}$ & $r$ & - & $\begin{array}{l}R \\
R \\
R\end{array}$ \\
\hline $\begin{array}{l}0.02 \\
0.01 \\
0.01 \\
0.01 \\
0.02 \\
\end{array}$ & $\begin{array}{l}+ \\
+ \\
-\end{array}$ & $\begin{array}{l}\underline{z} \\
\bar{z}\end{array}$ & $\begin{array}{l}+ \\
R \\
R \\
r \\
r\end{array}$ & $\begin{array}{l}+ \\
t \\
+ \\
+\end{array}$ & & $r$ & $\begin{array}{l}\mathrm{F} \\
\mathrm{F} \\
\mathrm{F} \\
\mathrm{F}\end{array}$ & & & $\begin{array}{l}\bar{z} \\
\bar{z} \\
\bar{z}\end{array}$ & & & & & & & $\begin{array}{l}\mathbf{R} \\
\mathbf{R} \\
\mathbf{R} \\
\mathbf{R}\end{array}$ & r & $\begin{array}{l}\mathrm{r} \\
\mathrm{R} \\
\mathrm{r} \\
\mathrm{r}\end{array}$ & $\begin{array}{l}R \\
R \\
r \\
r\end{array}$ & $\begin{array}{l}\mathrm{r} \\
\mathrm{r}\end{array}$ & $\begin{array}{l}\bar{z} \\
\overline{+}\end{array}$ & $\begin{array}{l}R \\
R \\
R \\
r\end{array}$ \\
\hline $\begin{array}{l}+ \\
+ \\
+ \\
+ \\
+\end{array}$ & $\bar{z}$ & $\begin{array}{l}- \\
+ \\
\mathrm{r} \\
\mathrm{r} \\
+\end{array}$ & $\begin{array}{l}\mathrm{r} \\
\mathbf{R} \\
\mathrm{r} \\
\mathrm{r} \\
\mathrm{R}\end{array}$ & $\begin{array}{l}r \\
R \\
r \\
r \\
r\end{array}$ & & $\begin{array}{l}r \\
r \\
r \\
r \\
R\end{array}$ & $\begin{array}{l}\mathrm{F} \\
\mathrm{F} \\
\mathrm{F} \\
\mathrm{F} \\
\mathrm{F}\end{array}$ & & & $\begin{array}{l} \pm \\
\pm \\
+\end{array}$ & & & & & & & $\begin{array}{l}\mathrm{R} \\
\mathrm{R} \\
\mathrm{R} \\
\mathrm{R} \\
\mathrm{R} \\
\end{array}$ & $\begin{array}{l}r \\
+ \\
r \\
r \\
r\end{array}$ & $\begin{array}{l}R \\
r \\
r \\
r \\
r \\
\end{array}$ & $\begin{array}{l}R \\
R \\
R \\
R \\
r \\
\end{array}$ & $\begin{array}{l}\mathbf{r} \\
\mathbf{R} \\
\mathbf{R} \\
\mathbf{r} \\
-\end{array}$ & $\begin{array}{l}\bar{z} \\
\bar{r}\end{array}$ & $\begin{array}{l}\mathrm{r} \\
\mathrm{r} \\
\mathrm{r} \\
\mathrm{r}\end{array}$ \\
\hline $\begin{array}{l}+ \\
+ \\
\mathrm{r} \\
+ \\
+\end{array}$ & & $\begin{array}{l}\mathrm{r} \\
\mathrm{r} \\
\mathrm{z}\end{array}$ & $\begin{array}{r}\mathrm{r} \\
-\end{array}$ & $\begin{array}{l}\mathrm{r} \\
\mathrm{r} \\
\mathrm{r} \\
\mathrm{r} \\
\mathrm{R}\end{array}$ & & $\begin{array}{l}\mathbf{R} \\
\mathbf{R} \\
\mathbf{R} \\
\mathbf{R} \\
\mathbf{R} \\
\end{array}$ & $\begin{array}{l}\mathrm{F} \\
\mathbf{R} \\
\mathrm{R} \\
\mathrm{R} \\
\mathrm{R} \\
\end{array}$ & & +1 & $\begin{array}{l}+ \\
t \\
\bar{r}\end{array}$ & & & & & & & $\begin{array}{l}R \\
R \\
r \\
R \\
\end{array}$ & $\begin{array}{l}\mathrm{r} \\
\mathrm{r} \\
\mathrm{r} \\
\mathrm{r} \\
\mathrm{r} \\
\end{array}$ & $\begin{array}{l}\mathrm{r} \\
\mathrm{r} \\
\mathrm{r} \\
\mathrm{R}\end{array}$ & $\begin{array}{l}\mathrm{r} \\
\mathrm{R} \\
\mathrm{R} \\
\mathrm{r} \\
\mathrm{R}\end{array}$ & $\begin{array}{l}+ \\
\mathrm{R} \\
\mathrm{r} \\
- \\
-\end{array}$ & $\begin{array}{l}\bar{z} \\
\bar{z}\end{array}$ & $\begin{array}{l}\frac{}{r} \\
\frac{r}{r}\end{array}$ \\
\hline $\begin{array}{l}+ \\
+\end{array}$ & & - & $r$ & $\begin{array}{l}\mathrm{r} \\
\mathrm{R}\end{array}$ & & $\begin{array}{r}\mathrm{R} \\
\mathrm{R} \\
1.00 \\
2.49 \\
0.90\end{array}$ & $\begin{array}{c}\mathrm{F} \\
\mathrm{F} \\
5.00 \\
4.92 \\
1.99\end{array}$ & & & $\begin{array}{l}r \\
r \\
r \\
r \\
r\end{array}$ & & & & & & & $\mathbf{R}$ & $\begin{array}{l}+ \\
\mathrm{r}\end{array}$ & $\begin{array}{l}R \\
R \\
r\end{array}$ & $\begin{array}{l}\mathbf{R} \\
\mathbf{R} \\
\mathbf{R} \\
\mathbf{R} \\
\mathbf{R}\end{array}$ & $\begin{array}{l}\bar{z} \\
\bar{z}\end{array}$ & $\begin{array}{l}+ \\
\pm \\
+ \\
+\end{array}$ & $\begin{array}{l}r \\
\mathrm{r} \\
+ \\
\mathrm{r} \\
+\end{array}$ \\
\hline & & & $\begin{array}{l}\mathrm{r} \\
\mathrm{r}\end{array}$ & $r$ & & $\begin{array}{c}4.02 \\
3.20 \\
3.40 \\
\text { F } \\
\text { F }\end{array}$ & $\begin{array}{c}1.02 \\
1.11 \\
1.09 \\
\mathrm{r} \\
\mathrm{r}\end{array}$ & & & $\begin{array}{l}\mathrm{r} \\
\mathrm{R} \\
\mathrm{R}\end{array}$ & & & & & & & $\begin{array}{l}\mathrm{R} \\
\mathrm{R} \\
\mathrm{R} \\
\mathrm{R}\end{array}$ & $\begin{array}{l}\mathrm{r} \\
\mathrm{r} \\
\mathrm{r} \\
\mathrm{r}\end{array}$ & $\begin{array}{l}\mathbf{R} \\
\mathbf{R} \\
\mathbf{R} \\
\mathbf{R}\end{array}$ & $\begin{array}{l}\mathbf{R} \\
\mathrm{r} \\
-\end{array}$ & - & $\begin{array}{l}+ \\
+ \\
+ \\
\mathrm{r}\end{array}$ & $\begin{array}{l}\mathrm{r} \\
\mathrm{R} \\
\mathrm{R} \\
\mathrm{R} \\
\end{array}$ \\
\hline \multirow[t]{2}{*}{+} & & & $\begin{array}{l}R \\
r\end{array}$ & $\begin{array}{l}r \\
r \\
r\end{array}$ & & $\begin{array}{l}\mathrm{F} \\
\mathrm{F} \\
\mathrm{F}\end{array}$ & $\mathrm{r}$ & $\begin{array}{l}\bar{z} \\
\bar{z}\end{array}$ & & $\begin{array}{l}\mathrm{R} \\
\mathrm{R} \\
\mathrm{R} \\
\mathrm{R}\end{array}$ & & & & & & & $\begin{array}{l}\mathrm{R} \\
\mathrm{R} \\
\mathrm{R}\end{array}$ & $\begin{array}{l}\mathrm{r} \\
\mathrm{r}\end{array}$ & $\begin{array}{l}\mathbf{R} \\
\mathbf{R} \\
\mathbf{R}\end{array}$ & + & & $\begin{array}{l}\mathbf{R} \\
\mathbf{R} \\
+\end{array}$ & $\begin{array}{l}R \\
r\end{array}$ \\
\hline & & & $\begin{array}{l}\mathbf{R} \\
\mathbf{R} \\
\mathbf{R}\end{array}$ & $\begin{array}{l}\mathrm{r} \\
\mathrm{r}\end{array}$ & & $\begin{array}{l}\mathrm{F} \\
\mathrm{F} \\
\mathrm{F}\end{array}$ & & $\begin{array}{l}+ \\
\pm \\
+\end{array}$ & & $\begin{array}{l}\mathbf{R} \\
\mathbf{R} \\
\mathbf{R}\end{array}$ & & & & & & & $\begin{array}{l}\mathrm{r} \\
\mathrm{r} \\
\mathrm{R}\end{array}$ & $\begin{array}{l}\mathrm{r} \\
\mathrm{r}\end{array}$ & $\begin{array}{l}\mathbf{R} \\
\mathbf{R} \\
\mathbf{R}\end{array}$ & $\begin{array}{l}\mathrm{r} \\
\mathrm{r} \\
\mathrm{r}\end{array}$ & & $\begin{array}{l}\mathrm{r} \\
\mathrm{R} \\
\mathrm{r}\end{array}$ & $\begin{array}{l}\mathbf{R} \\
\mathbf{R} \\
\mathbf{R}\end{array}$ \\
\hline
\end{tabular}


Table 2. Abundances of some radiolarians from Hole 503B. Symbols as for Table 1.

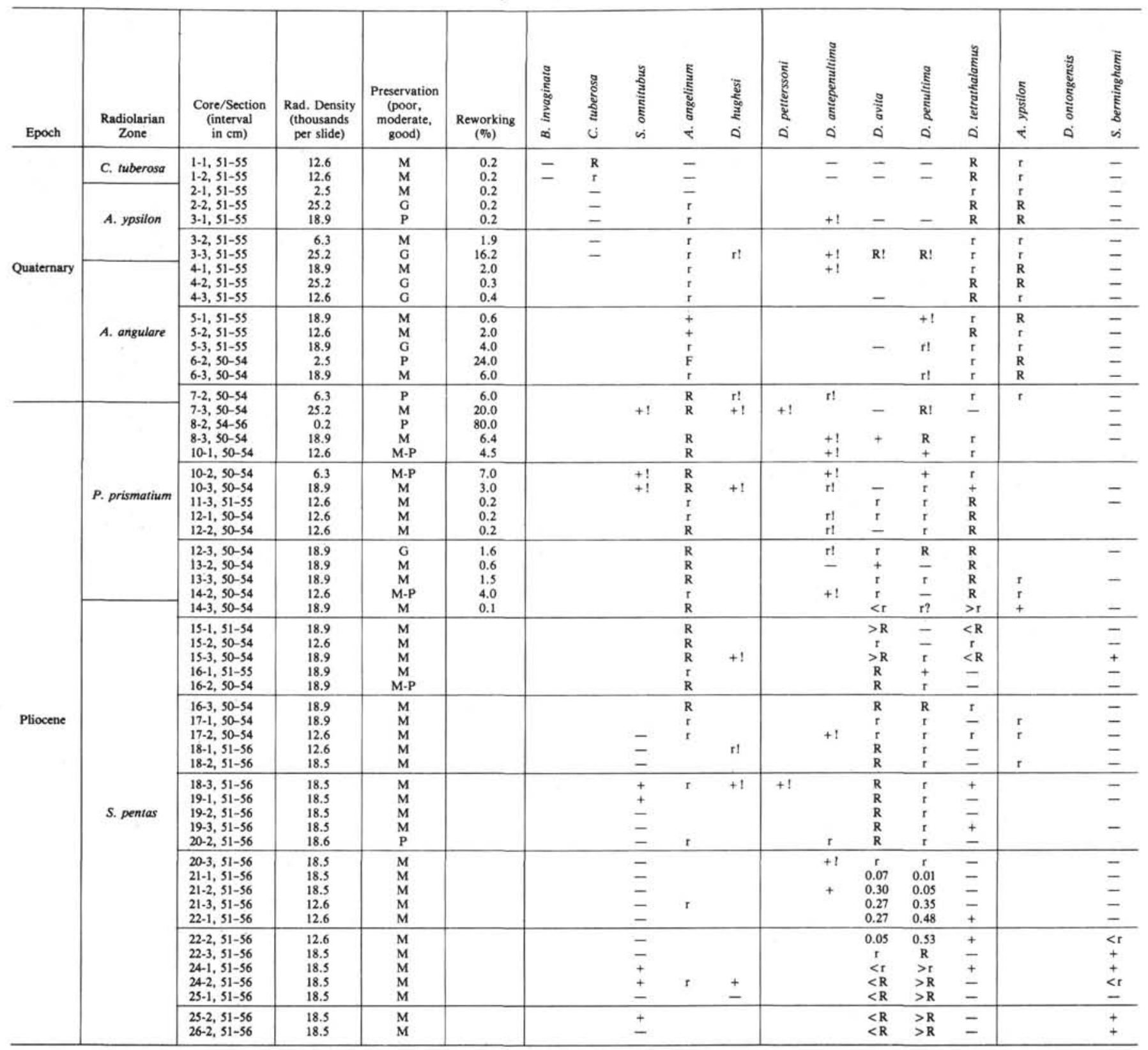

\section{SITE 502}

Results from land geology and from DSDP Legs 1, 4, 10 , and 15 have shown that radiolarians, and indeed siliceous microfossils in general, are not present in Tertiary sediments younger that about middle Miocene in the Gulf of Mexico and Caribbean (Riedel and Sanfilippo, 1973, Sanfilippo and Riedel, 1973, 1976).

Cores from Site 502 were examined for radiolarians to establish whether this absence is in fact continuous from the middle Miocene on and to shed some light on the reason for their absence. A channel sample of about 5-10 cc total volume was taken throughout the length of each section from Hole 502A, disaggregated, sieved through a mesh of $63 \mu \mathrm{m}$, and the coarse fraction acidified to remove the calcareous microfossils.
Siliceous radiolarian skeletons were found only in the top two cores (increasingly corroded downward), in Cores 502A-67 and 502A-68 (less corroded downward), and 502C-28-36. Those in Cores 502A-1 and 502A-2 are Quaternary, and those in 502A-67, 502A-68, and 502C28-36 are early late Miocene (Didymocyrtis antepenultima Zone). Many samples between these two intervals (notably from Cores 502A-50 and 51 and above) contain very rare fragmentary radiolarians and sponge spicules replaced by an opaque mineral.

The well-preserved radiolarians at the top and bottom of the section represent diverse assemblages characteristic of tropical, open ocean conditions, and as far as can be determined the opaque fragments are the remnants of a normal oceanic assemblage (including orosphaerids, Lamprocyclas, etc.). The evidence suggests 
Table 2. (Continued).

\begin{tabular}{|c|c|c|c|c|c|c|c|c|c|c|c|c|c|c|c|c|c|c|c|c|c|c|c|}
\hline $\begin{array}{l}\text { है } \\
\text { हूँ } \\
\text { जे }\end{array}$ & $\begin{array}{l}\text { g } \\
\text { v }\end{array}$ & $\begin{array}{l}\text { है } \\
\text { हू } \\
\text { जे }\end{array}$ & $\begin{array}{l}\mathscr{8} \\
\& \\
-3\end{array}$ & $\begin{array}{l}\text { वँँ } \\
\text { है }\end{array}$ & 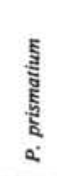 & 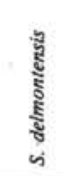 & 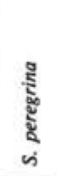 & 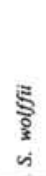 & 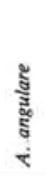 & 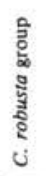 & 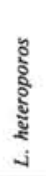 & 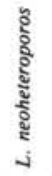 & 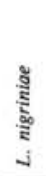 & 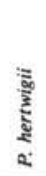 & 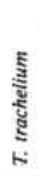 & 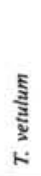 & 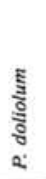 & 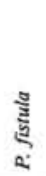 & $\begin{array}{l}\text { हू } \\
\text { s. } \\
\text { ú }\end{array}$ & 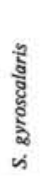 & हू हू & 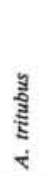 & 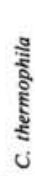 \\
\hline $\begin{array}{l}\bar{z} \\
\overline{+1} \\
-\end{array}$ & $\begin{array}{l}\mathbf{R} \\
\mathbf{R} \\
\mathrm{R} \\
\mathrm{R} \\
\mathrm{r} \\
\end{array}$ & & $\frac{\mathrm{r}}{\mathrm{g}}$ & & & $r !$ & $\begin{array}{l}\mathrm{r} ! \\
\mathrm{r} ! \\
\mathrm{r} ! \\
\mathrm{r} ! \\
\mathrm{r} !\end{array}$ & & $\begin{array}{l}- \\
-\end{array}$ & & $\overline{-}$ & $\begin{array}{l}\bar{z} \\
\bar{z}\end{array}$ & $\begin{array}{l}\mathrm{R} \\
\mathrm{R} \\
\mathrm{R} \\
\mathrm{R}\end{array}$ & $\frac{\bar{r}}{r}$ & $\begin{array}{l}r \\
r \\
r \\
R\end{array}$ & $\underline{-}$ & & & & $\underline{r}$ & $\frac{\bar{R}}{\overline{+}}+$ & & $\frac{r}{-}$ \\
\hline $\begin{array}{l}\bar{z} \\
\bar{z}\end{array}$ & $\begin{array}{l}\mathbf{R} \\
\mathbf{R} \\
\mathbf{F} \\
\mathrm{F} \\
\mathbf{R} \\
\end{array}$ & & $\begin{array}{l}\mathrm{r} \\
\mathrm{R} \\
\mathrm{r}\end{array}$ & & $\begin{array}{l}+1 \\
=\end{array}$ & $\begin{array}{l}\mathrm{r} ! \\
\mathrm{R} ! \\
\mathrm{r} !\end{array}$ & $\begin{array}{l}\mathrm{r} ! \\
\mathbf{R} ! \\
\mathbf{R} ! \\
+! \\
+!\end{array}$ & & $\begin{array}{l}- \\
\overline{+} \\
+ \\
\text { R }\end{array}$ & . & $\begin{array}{l}- \\
-\end{array}$ & $\begin{array}{l}\overline{-} \\
+ \\
t \\
\text { t }\end{array}$ & $\begin{array}{l} \\
r \\
+ \\
r \\
+\end{array}$ & $\begin{array}{l}+ \\
+ \\
+ \\
+\end{array}$ & $\begin{array}{l}\mathbf{R} \\
\mathrm{R} \\
\mathrm{R} \\
\mathrm{R} \\
\mathrm{R}\end{array}$ & $\begin{array}{l}\overline{+1} \\
\overline{-} \\
-\end{array}$ & $\begin{array}{l} \pm 1 \\
=\end{array}$ & & & \pm & $\begin{array}{l}\overline{ \pm} \\
\pm\end{array}$ & & $\begin{array}{l}+ \\
\mathrm{r} \\
\mathrm{r}\end{array}$ \\
\hline $\begin{array}{l}\bar{z} \\
\bar{z}\end{array}$ & $\begin{array}{l}\mathbf{R} \\
\mathbf{R} \\
\mathbf{R} \\
\mathbf{R} \\
\mathbf{F}\end{array}$ & & $\mathrm{r}$ & & $\begin{array}{c}+! \\
0.01 ! \\
= \\
=\end{array}$ & $\begin{array}{l}\mathrm{r} ! \\
\mathrm{r} ! \\
\mathrm{R} ! \\
\mathrm{R} !\end{array}$ & $\begin{array}{l}\mathrm{r} ! \\
\mathrm{r} ! \\
\mathbf{R} ! \\
\mathbf{R} ! \\
\mathbf{R} !\end{array}$ & & $\begin{array}{l}+ \\
+ \\
\frac{r}{r}\end{array}$ & & $\begin{array}{l}- \\
- \\
+ \\
r \\
<r\end{array}$ & $\begin{array}{l}+\stackrel{+}{R} \\
\frac{r}{r} \\
>r\end{array}$ & $\begin{array}{l}\bar{z} \\
\bar{z}\end{array}$ & $\begin{array}{l}\bar{z} \\
\bar{r}\end{array}$ & $\begin{array}{l}R \\
+ \\
r \\
-\end{array}$ & $\begin{array}{l}+ \\
F \\
F \\
F \\
F\end{array}$ & $\begin{array}{l}- \\
+1 \\
+1 \\
+1 \\
+1\end{array}$ & & +1 & $\begin{array}{l}\bar{r} \\
+ \\
\overline{-}\end{array}$ & + & +1 & $\begin{array}{l}+ \\
r \\
r \\
r\end{array}$ \\
\hline $\begin{array}{l}\overline{+} \\
+ \\
-\end{array}$ & $\begin{array}{l}\mathrm{r} \\
\mathrm{R} \\
\mathrm{R} \\
\mathrm{R}\end{array}$ & & + & + & $\begin{array}{l}\overline{+1} \\
\overline{+1} \\
0.01 ! \\
0.011\end{array}$ & $\begin{array}{l}\mathbf{R} ! \\
\mathbf{R} ! \\
+ \\
\mathbf{R} ! \\
\mathrm{r} !\end{array}$ & $\begin{array}{l}\mathbf{R} ! \\
\mathbf{R} ! \\
\mathbf{R} ! \\
\mathbf{R} ! \\
\mathbf{R} !\end{array}$ & & $\begin{array}{l}\bar{z} \\
\bar{z}\end{array}$ & $\begin{array}{l}+1 \\
+1\end{array}$ & $\begin{array}{c}++ \\
R \\
>r \\
R\end{array}$ & $\begin{array}{l}r \\
r \\
<r \\
r\end{array}$ & $\bar{z}$ & $\bar{z}$ & $\overline{\bar{z}}$ & $\begin{array}{l}\mathrm{F} \\
\mathrm{F} \\
\mathrm{F} \\
\mathrm{F}\end{array}$ & $\begin{array}{l}+! \\
t ! \\
+! \\
= \\
-\end{array}$ & & & + & $\begin{array}{l}- \\
+ \\
+\end{array}$ & & + \\
\hline $\begin{array}{l}\overline{+} \\
\mathrm{r} \\
+\end{array}$ & $\begin{array}{l}\mathbf{F} \\
\mathbf{R} \\
\mathbf{R} \\
r \\
\mathbf{R} \\
\end{array}$ & & $\begin{array}{l}r \\
r \\
r \\
r \\
r\end{array}$ & $\begin{array}{l}I \\
= \\
=\end{array}$ & $\begin{array}{l}\mathrm{r} \\
+ \\
\mathrm{r} \\
\mathrm{r} \\
\mathrm{R}\end{array}$ & $\begin{array}{l}\mathrm{r} ! \\
\mathrm{r} ! \\
\mathrm{r} ! \\
\mathrm{r} ! \\
\mathrm{r} !\end{array}$ & $\begin{array}{l}\mathrm{R} ! \\
\mathrm{R} ! \\
\mathrm{r} ! \\
\mathrm{r} ! \\
\mathrm{r} !\end{array}$ & & & $r !$ & $\begin{array}{l}>r \\
>r \\
>r \\
R \\
r\end{array}$ & $\begin{array}{l}<r \\
<r \\
<r \\
r \\
r\end{array}$ & & & $\overline{-}$ & $\begin{array}{l}\mathrm{F} \\
\mathrm{R} \\
\mathrm{R} \\
\mathrm{R} \\
\mathrm{R}\end{array}$ & $\begin{array}{l}- \\
+! \\
\mathrm{r} ! \\
\mathrm{r} ! \\
\mathrm{r} !\end{array}$ & & $\begin{array}{l}+1 \\
+1\end{array}$ & $\begin{array}{l}\mathrm{r} \\
\mathrm{r} \\
\mathrm{r} \\
\mathrm{r} \\
\mathrm{r}\end{array}$ & $\stackrel{r}{r}$ & & $\begin{array}{l}+ \\
\mathrm{r} \\
\mathrm{r} \\
\mathrm{r}\end{array}$ \\
\hline $\begin{array}{l} \pm \\
\pm \\
+\end{array}$ & $\begin{array}{l}\mathbf{R} \\
\mathbf{R} \\
\mathbf{R} \\
\mathrm{r} \\
\mathbf{R}\end{array}$ & & $\begin{array}{l}+ \\
\mathbf{r} \\
\mathbf{r} \\
+ \\
-\end{array}$ & $\begin{array}{l}\bar{z} \\
\bar{z}\end{array}$ & $\begin{array}{l}\mathrm{r} \\
\mathrm{r} \\
\mathrm{R} \\
\mathrm{r}\end{array}$ & $\begin{array}{c}\mathrm{r} ! \\
0.01 ! \\
0.02 ! \\
0.18 ! \\
0.05 !\end{array}$ & $\begin{array}{l}\mathrm{r} ! \\
0.03 ! \\
0.05 ! \\
0,37 ! \\
4.4\end{array}$ & & & & $\begin{array}{l}+ \\
\mathbf{R} \\
+ \\
\mathbf{R} \\
\mathrm{R}\end{array}$ & $\begin{array}{l}\overline{+} \\
\bar{r} \\
\overline{-}\end{array}$ & & & & $\begin{array}{l}R \\
R \\
R \\
R \\
R \\
r\end{array}$ & $\begin{array}{l}\overline{+1} \\
\overline{-} \\
=\end{array}$ & - & & $\begin{array}{l}r \\
r \\
r \\
R \\
R\end{array}$ & $\underline{\mathrm{r}}$ & & $\begin{array}{l}r \\
+ \\
R \\
r \\
R\end{array}$ \\
\hline$\frac{\bar{r}}{\bar{r}}$ & $\begin{array}{l}\mathbf{R} \\
\mathbf{R} \\
\mathbf{R} \\
\mathbf{R} \\
\mathbf{R}\end{array}$ & & $\begin{array}{l}\mathrm{r} \\
\mathrm{r} \\
\mathrm{r} \\
\mathrm{r} \\
\mathrm{r}\end{array}$ & $\begin{array}{l}\bar{z} \\
\bar{z}\end{array}$ & $\begin{array}{l}r \\
r \\
r \\
+ \\
+\end{array}$ & $\begin{array}{l}= \\
=\end{array}$ & $\begin{array}{c}\mathbf{F} \\
\mathbf{F} \\
\mathrm{F} \\
\mathrm{F} \\
\mathrm{F}\end{array}$ & & & & $\begin{array}{l}\mathrm{r} \\
\mathrm{R} \\
\mathrm{r} \\
- \\
-\end{array}$ & $\begin{array}{l}\bar{z} \\
\bar{z}\end{array}$ & $=$ & & & $\begin{array}{l}\mathbf{R} \\
\mathbf{R} \\
\mathbf{R} \\
\mathrm{R} \\
\mathrm{R}\end{array}$ & $\begin{array}{l}+1 \\
+1 \\
- \\
-\end{array}$ & $\begin{array}{l}\bar{z} \\
\overline{+} \\
\overline{-}\end{array}$ & & $\begin{array}{l}\mathrm{r} \\
\mathrm{r} \\
\mathrm{R} \\
\mathrm{r} \\
+ \\
\end{array}$ & & & $\begin{array}{l}r \\
r \\
r \\
r \\
+\end{array}$ \\
\hline $\begin{array}{l}+ \\
t \\
t \\
r \\
t\end{array}$ & $\begin{array}{l}R \\
R \\
R \\
R \\
F \\
R\end{array}$ & & $\begin{array}{l}\mathrm{r} \\
\mathrm{r} \\
\mathrm{R} \\
\mathrm{r}\end{array}$ & $\begin{array}{l}\bar{z} \\
\bar{z}\end{array}$ & $\begin{array}{l}\mathrm{r} \\
\mathrm{R} \\
\mathrm{r} \\
\mathrm{r}\end{array}$ & $\begin{array}{l}\bar{z} \\
\bar{z}\end{array}$ & $\begin{array}{l}\mathrm{F} \\
\mathrm{F} \\
\mathrm{F} \\
\mathrm{F} \\
\mathrm{F}\end{array}$ & & & & $\begin{array}{l}\bar{z} \\
\bar{z}\end{array}$ & $\begin{array}{l}\bar{z} \\
\bar{z}\end{array}$ & $\frac{+}{-}$ & & & $\begin{array}{l}\mathrm{R} \\
\mathrm{R} \\
\mathrm{R} \\
\mathrm{R} \\
\mathrm{R}\end{array}$ & $\begin{array}{l}+1 \\
\bar{r} \\
+1\end{array}$ & $\begin{array}{l}\mathbf{r} \\
+ \\
\mathbf{r} \\
\mathbf{R} \\
-\end{array}$ & & $\begin{array}{l}r \\
r \\
R \\
R \\
R \\
\end{array}$ & & & $\begin{array}{l}+ \\
\mathbf{R} \\
\mathbf{R} \\
\mathbf{R} \\
\mathrm{r}\end{array}$ \\
\hline $\begin{array}{l}+ \\
\mathrm{r} \\
0.01 \\
0.01 \\
0.02\end{array}$ & $\begin{array}{c}R \\
R \\
0.02 \\
0.26 \\
0.06\end{array}$ & & $\begin{array}{l}\mathrm{r} \\
\mathrm{R} \\
\mathrm{R} \\
+ \\
- \\
\end{array}$ & $\begin{array}{l} \pm \\
\pm \\
+\end{array}$ & $\begin{array}{l}r \\
r \\
r \\
r \\
r\end{array}$ & - & $\begin{array}{l}\mathbf{F} \\
\mathbf{F} \\
\mathbf{F} \\
\mathrm{F} \\
\mathrm{F}\end{array}$ & & & & & & & & & $\begin{array}{l}\mathbf{R} \\
\mathbf{R} \\
\mathbf{R} \\
\mathbf{R} \\
\mathbf{R}\end{array}$ & $\begin{array}{l}+1 \\
r ! \\
+1 \\
R\end{array}$ & $\begin{array}{l}\mathrm{r} \\
+ \\
\mathrm{r} \\
\mathrm{R} \\
\end{array}$ & & $\begin{array}{l}r \\
r \\
r \\
R \\
R \\
\end{array}$ & $\begin{array}{l}+ \\
+\end{array}$ & & $\begin{array}{l}\mathbf{r} \\
\mathbf{R} \\
\mathbf{R} \\
\mathbf{R} \\
\mathbf{R}\end{array}$ \\
\hline $\begin{array}{l}0.22 \\
0.45 \\
0.04 \\
0.48 \\
R\end{array}$ & $\begin{array}{l}0.01 \\
0.01 \\
= \\
=\end{array}$ & - & $\begin{array}{l}+ \\
\mathrm{r} \\
\mathrm{R} \\
\mathrm{r} \\
\mathrm{R}\end{array}$ & $\begin{array}{l}+ \\
+ \\
+ \\
+\end{array}$ & $\begin{array}{l}\mathrm{r} \\
+ \\
\mathrm{r} \\
\mathrm{r} \\
\mathrm{r}\end{array}$ & $\begin{array}{l}\bar{z} \\
\bar{z}\end{array}$ & $\begin{array}{l}\mathrm{F} \\
\mathrm{F} \\
\mathrm{F} \\
\mathrm{F} \\
\mathrm{F}\end{array}$ & & & & & & & & & $\begin{array}{l}r \\
R \\
R \\
R \\
R\end{array}$ & $\begin{array}{l} \\
R \\
R \\
R \\
R \\
R\end{array}$ & $\begin{array}{l}- \\
+ \\
r \\
r \\
r\end{array}$ & & $\begin{array}{l}\mathbf{R} \\
R \\
R \\
R \\
R \\
R\end{array}$ & + & & $\begin{array}{l}\mathbf{R} \\
\mathbf{R} \\
\mathbf{R} \\
\mathbf{R} \\
\mathbf{R}\end{array}$ \\
\hline $\begin{array}{c}>r \\
r \\
r \\
>r \\
r\end{array}$ & $\begin{array}{l}\bar{z} \\
\bar{z}\end{array}$ & $\begin{array}{l}- \\
\bar{r} \\
\mathrm{r} \\
+\end{array}$ & $\begin{array}{l}R \\
r \\
r \\
r \\
r \\
\end{array}$ & $\begin{array}{l}\mathrm{r} \\
\mathrm{r} \\
+ \\
+ \\
\mathrm{r}\end{array}$ & $\begin{array}{l} \\
R \\
R \\
r \\
r \\
-\end{array}$ & $\begin{array}{l}\mathbf{R} \\
+ \\
\mathbf{R} \\
+ \\
\mathbf{r}\end{array}$ & $\begin{array}{l}\mathrm{F} \\
\mathrm{F} \\
\mathrm{F} \\
\mathrm{F} \\
\mathrm{F}\end{array}$ & & & & & & & & & $\begin{array}{l}R \\
R \\
r \\
r \\
r\end{array}$ & $\begin{array}{l}\mathbf{R} \\
\mathrm{r} \\
\mathbf{R} \\
\mathbf{R} \\
\mathbf{R}\end{array}$ & $\begin{array}{l}r \\
+ \\
+ \\
+ \\
+\end{array}$ & & $\begin{array}{l} \\
R \\
R \\
R \\
R \\
R\end{array}$ & $\begin{array}{l}r \\
+\end{array}$ & $=$ & $\begin{array}{l}\mathbf{R} \\
\mathbf{R} \\
\mathbf{R} \\
\mathbf{F} \\
\mathbf{R}\end{array}$ \\
\hline $\begin{array}{l}\mathrm{r} \\
\mathrm{r}\end{array}$ & $\bar{z}$ & $\mp$ & $\stackrel{r}{r}$ & ${ }_{r}^{r}$ & $\overline{-}$ & + & $\begin{array}{l}F \\
F\end{array}$ & & & & & & & & & $\begin{array}{l}\mathrm{r} \\
\mathrm{r}\end{array}$ & $\begin{array}{l}\mathrm{F} \\
\mathrm{R}\end{array}$ & $+\underset{r}{+}$ & & $\mathrm{r}$ & $\mathrm{r}$ & $\overline{-}$ & $\begin{array}{l}\mathbf{R} \\
\mathbf{R}\end{array}$ \\
\hline
\end{tabular}

that the absence of siliceous microfossils in sediments of this region is continuous from the early late Miocene to the Quaternary and that dissolution within the sediment (rather than the absence of specimens in the waters above) was responsible.

Hole 502A reached only the uppermost level of occurrence of Miocene siliceous microfossils, but Hole 502C was cored deeper and might therefore provide an indication as to whether radiolarians occur continuously or intermittently in the sediment column below the lower upper Miocene. Few, moderately well-preserved radiolarians, which correspond to those in Core 502A-68, occur in Cores 502C-28 and 29. Below this, the abundance and state of preservation decline to a minimum in Core 502C-35. Their preservation improves within a pyritized mass in Core 502C-36, but they, remain very rare as a result of dilution with pyroclastic components. The assemblage in this sample still represents the $D$. antepenultima Zone, but it is possible that the lump was displaced from higher in the hole and that the sediment at this depth is somewhat older. However that may be, the worsening preservation in Cores 502C-30-35 indicates that the transition from radiolarian-rich sediment in the middle Tertiary to lack of radiolarians in upper Tertiary sediment is irregular. It is sufficient to account for the differences in age (ranging from early to early late Miocene) of the youngest radiolarian sediments at the various previous drilling sites, which have been more or less discontinuously cored.

The radiolarian fauna of Cores 502A-62 and 502C-28 to $502 \mathrm{C}-36$ includes the following species (range of estimated percentage abundances relative to the entire as- 

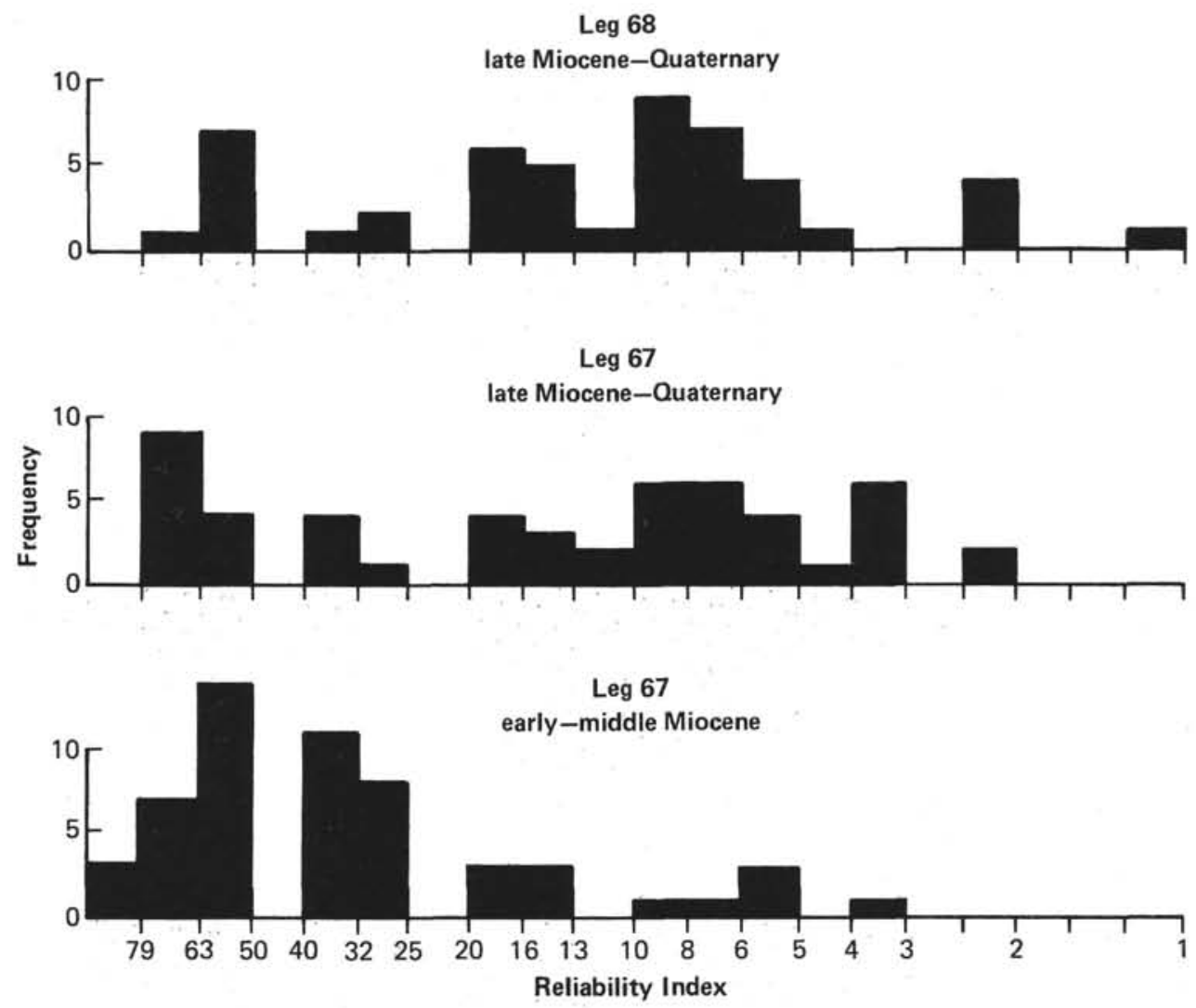

Figure 1. Frequency of use of reliability index values. Each histogram represents reliability scores of approximately 50 events. The reliability index values are plotted on an antilog scale (see text).

semblage): Diartus hughesi, $0-8 \%$; D. petterssoni, 0 $2 \%$; Didymocyrtis antepenultima, $0-0.2 \% ; D$. laticonus, 0-2\%; Lithopera bacca, 0-0.1\%; Lychnocanoma audax, $0-0.1 \%$; Stichocorys delmontensis, $0.1-13 \% ; S$. peregrina, 0-1\%; Phormostichoartus corbula, 0-5\%; P. doliolum, $0-0.1 \%$; P. fistula, $0-0.1 \%$.

\section{SITE 503}

Sediments at Site 503 represent an almost complete undisturbed record from the upper Miocene through the Quaternary. Radiolarians are well preserved and abundant throughout Holes 503A and 503B, except for being somewhat corroded and sparse in several cores near the Plio/Pleistocene boundary.

Reworking of Miocene radiolarians into younger sediments is indicated in samples from Cores 503A-1 through 503A-29 and 503B-1 through 503B-13. The reworking is generally minor but approaches $20 \%$ in samples from Cores 503A-4, 7, 10, 11, and 503B-3, 6, 7; and more than $50 \%$ in Cores 503A-7, 503A-9, and 503B-8.

The top of the Pterocanium prismatium Zone, which is considered to be approximately the Pliocene/Pleistocene boundary, is placed between Samples 503A-7-2, $50-54 \mathrm{~cm}$ and 503B-7-3, 50-54 cm in both Holes 503A and 503B. The evolutionary transition from Spongaster berminghami to $S$. pentas, approximating the Miocene/ Pliocene boundary, occurs between Samples 503A-31-3, $50-54 \mathrm{~cm}$ and $503 \mathrm{~A}-34-2,50-54 \mathrm{~cm}$.

The Pliocene radiolarian zones, $P$. prismatium and $S$. pentas, are well represented in both holes. Hole 503A penetrated the upper Miocene Didymocyrtis penultima Zone and reached the top of the $D$. antepenultima Zone ( 7.8 m.y.).

One pair of events-namely, the earliest morphotypic occurrence of $P$. prismatium and the evolutionary transition from $S$. berminghami to $S$. pentas-occur in the order described by Sanfilippo and Riedel (1975) for DSDP Sites 82 and 84 and represent a minor inconsistency with that reported by Theyer et al. (1978) for a single core taken farther west in the tropical Pacific. We believe this to be due to diachroneity of the earliest occurrence of $P$. prismatium rather than of the evolutionary transition from $S$. berminghami to $S$. pentas.

Abundances of radiolarians at Site 503 are recorded in Tables 1 and 2.

\section{CORRELATIONS}

Figure 2 shows the correlations between Holes 503A and 503B and with Site $495\left(12^{\circ} 29.8^{\prime} \mathrm{N}, 91^{\circ} 02.3^{\prime} \mathrm{W}\right)$, the "oceanic reference" drilled on Leg 67. Most of the correlation lines present no problem, but there are a few conflicts which the reliability index scores (see Procedures) in Table 3 help to resolve. This assumes that there are no real biogeographic differences in the order of events at the two sites.

The order of the evolutionary transitions from Spongaster pentas to $S$. tetras and Didymocyrtis penultima to D. avita (Events 17 and 18), is reversed at Site 495 compared to Site 503, and the reliability indices indicate that the order at Site 503 is more likely correct. 


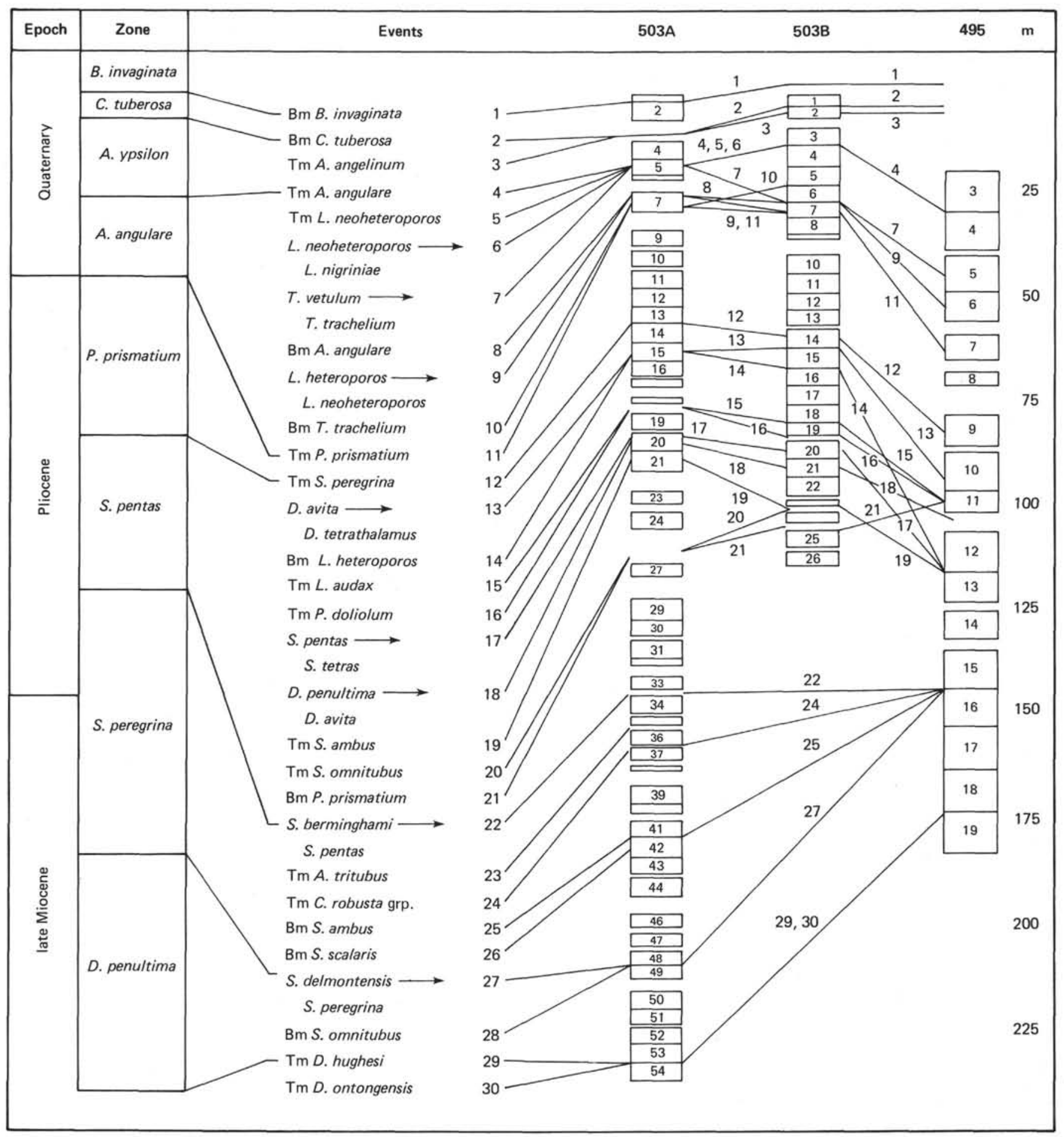

Figure 2. Correlation of radiolarian events in Holes 503A and 503B of Leg 68 and Hole 495 of Leg 67. Each rectangular box represents a core with its number shown inside. Numbers labeling correlation lines correspond to the radiolarian events listed to the left. (Sample intervals bracketing events and a reliability index for each event are given in Table 3. )

The discrepancy between the relative position of the lower limit of Lamprocyrtis heteroporos (Event 14) at the two sites is resolved, by the reliability indices, in favor of that at Site 503 .

The lower limit of Pterocanium prismatium (Event 21) occurs inconsistently high in the sequence at Site 495 , but this is not surprising in view of its low index of reliability due to low abundance and intermittent occurrence (Westberg and Riedel, in press).

The lower limit of Theocorythium trachelium occurs higher in Hole 503B than in Hole 503A. The event has the same low index of reliability in both sequences and therefore the lower occurrence is considered more likely correct. 
Table 3. Magnetic epochs and radiolarian zones and events at Sites 503 and 495 . The number below the sample numbers is the index of reliability (see text). Events are serially numbered for comparison with Figure 2.

\begin{tabular}{|c|c|c|c|c|c|}
\hline Magnetic Epoch & $\begin{array}{l}\text { Radiolarian } \\
\text { Zone }\end{array}$ & $\begin{array}{l}\text { Radiolarian } \\
\text { Event }\end{array}$ & $503 \mathrm{~A}$ & 503B & 495 \\
\hline \multirow{7}{*}{ Brunhes } & B. invaginata & \multirow[b]{2}{*}{$1 \mathrm{Bm} \mathrm{B}$. invaginata } & $1-1,50-52$ & & \\
\hline & \multirow[t]{2}{*}{ C. tuberosa } & & $2-1,100-102$ & & \\
\hline & & \multirow[b]{2}{*}{$2 \mathrm{Bm}$ C. tuberosa } & $2-3,50-52$ & $1-2,51-55$ & \\
\hline & \multirow{4}{*}{ A. ypsilon } & & $\begin{array}{c}4-1,50-54 \\
28\end{array}$ & $\begin{array}{c}2-1,51-55 \\
38\end{array}$ & \\
\hline & & \multirow[b]{2}{*}{$3 \mathrm{Tm}$ A. angelinum } & $2-3,50-52$ & $2-1,51-55$ & \\
\hline & & & $\begin{array}{c}4-1,50-54 \\
56\end{array}$ & $\begin{array}{l}2-2,51-55 \\
56\end{array}$ & \\
\hline & & \multirow[b]{2}{*}{$4 \operatorname{Tm} A$. angulare } & $5-1,50-54$ & $3-3,51-55$ & $3-6,20-22$ \\
\hline \multirow{14}{*}{ Matuyama } & \multirow{13}{*}{ A. angulare } & & $\begin{array}{c}5-2,50-54 \\
9\end{array}$ & $\begin{array}{c}4-1,51-55 \\
9\end{array}$ & $\begin{array}{c}4-1,75-77 \\
2\end{array}$ \\
\hline & & \multirow[b]{2}{*}{$5 \mathrm{Tm}$ L. neoheteroporus } & $5-1,50-54$ & $3-3,51-55$ & $4-1,75-77$ \\
\hline & & & $5-2,50-54$ & $4-1,51-55$ & $\begin{array}{c}4-3,75-77 \\
14\end{array}$ \\
\hline & & $\begin{array}{l}{ }^{6} \begin{array}{l}\text { L. neoheteroporos } \\
\text { L. nigriniae }\end{array} \\
\end{array}$ & $\begin{array}{l}5-150-54 \\
5-2,50-54 \\
9\end{array}$ & $\begin{array}{c}3-3,51-55 \\
4-3,51-55 \\
9\end{array}$ & \\
\hline & & \multirow{2}{*}{$\begin{array}{l}7 . \text { vetulum } \longrightarrow \\
T . \text { trachelium }\end{array}$} & $5-1,50-54$ & $6-2,50-54$ & $5-2,40-42$ \\
\hline & & & $\begin{array}{c}5-2,50-54 \\
9\end{array}$ & $7-2,50-54$ & $5-5,40-42$ \\
\hline & & \multirow[b]{2}{*}{$8 \mathrm{Bm} \mathrm{A}$. angulare } & $7-1,50-54$ & $6-3,50-54$ & $6-4,10-12$ \\
\hline & & & $\begin{array}{c}7-2,50-54 \\
6\end{array}$ & $\begin{array}{c}7-2,50-54 \\
2\end{array}$ & $6-\mathrm{CC},{ }_{2}^{3-4}$ \\
\hline & & \multirow{2}{*}{$\begin{array}{l}9 \text { L. heteroporos - } \\
\text { L. neoheteroporos }\end{array}$} & $7-1,50-54$ & $7-2,50-54$ & \\
\hline & & & $7-2,50-54$ & $7-3,50-54$ & \\
\hline & & \multirow[b]{2}{*}{$10 \mathrm{Bm} T$. trachelium } & $7-2,50-54$ & $5-3,51-55$ & \\
\hline & & & $\begin{array}{c}7-3,50-54 \\
5\end{array}$ & $\begin{array}{c}6-2,50-54 \\
5\end{array}$ & \\
\hline & & \multirow[b]{2}{*}{$11 \mathrm{Tm}$ P. prismatium } & $7-2,50-54$ & $7-2,50-54$ & $7-1,70-72$ \\
\hline & \multirow{2}{*}{ P. prismatium } & & $\begin{array}{c}7-3,50-54 \\
2\end{array}$ & $7 \cdot 3,50-54$ & $\begin{array}{c}7-3,70-72 \\
9\end{array}$ \\
\hline & & & $13-3,50-54$ & $14-2,50-54$ & $9-3,75-77$ \\
\hline & & $12 \mathrm{Tm} S$. peregrina & $\begin{array}{c}14-3,50-54 \\
56\end{array}$ & $\begin{array}{c}14-3,50-54 \\
56\end{array}$ & $\begin{array}{c}9-6,26-28 \\
75\end{array}$ \\
\hline & & & $15-2,66-70$ & $14-3,50-54$ & $6-1,92-94$ \\
\hline Gauss & & $\begin{array}{l}13 \text { D. avita } \overrightarrow{\text { D. tetrathalamus }} \\
\end{array}$ & $15-3,50-54$ & $\begin{array}{c}15-1,50-54 \\
13\end{array}$ & $11-1,60-62$ \\
\hline & & & $15-2,66-70$ & $15-3,50-54$ & $13-5,54-55$ \\
\hline & & $14 \mathrm{Bm} \mathrm{L}$. heteroporos & $\begin{array}{c}15-3,50-54 \\
6\end{array}$ & $\begin{array}{c}16-1,51-55 \\
13\end{array}$ & $\begin{array}{c}14-1,30-32 \\
9\end{array}$ \\
\hline & & & $16-2,84-88$ & $18-3,51-56$ & $11-1,60-62$ \\
\hline & & $15 \operatorname{Tm}$ L. audax & $\begin{array}{c}19-2,60-64 \\
7\end{array}$ & $19-1,51-56$ & $11-3,60-62$ \\
\hline & & & $16-2,84-88$ & $19-3,51-56$ & $11-3,60-62$ \\
\hline & & $16 \mathrm{Tm} P$. doliolum & $\begin{array}{c}19-2,60-64 \\
19\end{array}$ & $\begin{array}{c}20-2,51-56 \\
19\end{array}$ & ${ }_{11}^{11-C_{1}, 4-6}$ \\
\hline & & & $20-1,50-54$ & $20-2,51-56$ & $\frac{19}{12-7,31-33}$ \\
\hline & S. pentas & $\begin{array}{l}17 \text { S. pentas }- \\
\text { S. tetras }\end{array}$ & $20-3,50-54$ & $\begin{array}{c}20-3,51-56 \\
50\end{array}$ & $13-5,54-55$ \\
\hline & & $\begin{array}{l}18 \text { D. penultima - } \\
\text { D. avita }\end{array}$ & $\begin{array}{c}20-2,50-54 \\
20-3,50-54 \\
2\end{array}$ & $\begin{array}{c}21-2,51-56 \\
21-3,51-56 \\
4\end{array}$ & $\begin{array}{c}11-3,60-62 \\
12-3,30-32 \\
4\end{array}$ \\
\hline & & & $21-2,50-54$ & $22-3,51-56$ & $\frac{4}{12-7,31-33}$ \\
\hline & & $19 \mathrm{Tm}$ S. ambus & $21-3,50-54$ & $24-1,51-56$ & $13-5,54-55$ \\
\hline & & & $24-3,50-54$ & $22-3,51-56$ & \\
\hline & & $20 \mathrm{Tm}$ S. omnitubus & $29-1, \frac{50-54}{5}$ & $24-1,51-56$ & \\
\hline & & & $24-3,50-54$ & $24-2,51-56$ & $11-3,60-62$ \\
\hline & & $21 \mathrm{Bm} P$. prismatium & $\begin{array}{c}29-1,50-54 \\
9\end{array}$ & $\begin{array}{c}25-1,51-56 \\
19\end{array}$ & ${ }_{11}^{11-C C}, 4-6$ \\
\hline & & S. berminghami - & $31-3,50-54$ & & $15-5,30-32$ \\
\hline & & $\begin{array}{l}22 \\
\text { S. perming }\end{array}$ & $\begin{array}{c}34-2,50-54 \\
19\end{array}$ & & $\begin{array}{c}16-2,30-32 \\
38\end{array}$ \\
\hline Gilbert & & & $34-3,50-54$ & & \\
\hline & & $23 \mathrm{Tm}$ A. tritubus & $\begin{array}{c}36-1,81-84 \\
2\end{array}$ & & \\
\hline & & & $36-2,50-54$ & & $15-5,30-32$ \\
\hline & S. peregrina & $24 \mathrm{Tm}$ C. robusta group & $\begin{array}{c}37-1,50-54 \\
11\end{array}$ & & $\begin{array}{c}16-2,30-32 \\
50\end{array}$ \\
\hline & & & $41-3,50-54$ & & $15-5,30-32$ \\
\hline & & $25 \mathrm{Bm} \mathrm{S.} \mathrm{ambus}$ & $42-1,50-54$ & & $16-2, \frac{30-32}{9}$ \\
\hline & & & $42-1,50-54$ & & \\
\hline & & $26 \mathrm{Bm} S$. scalaris & $42-2,50-54$ & & \\
\hline & & S. delmontensis - & $48-2,50-54$ & & $15-1,30-32$ \\
\hline & & $\begin{array}{l}27 \text { S. deimontensis - } \\
\text { S. peregrina }\end{array}$ & $\begin{array}{c}49-1,50-54 \\
25\end{array}$ & & $\begin{array}{c}16-2,30-32 \\
37\end{array}$ \\
\hline & D. penultima & $28 \mathrm{Bm}$ S. omnitubus & $\begin{array}{c}48-2,50-54 \\
49-1,50-54 \\
19\end{array}$ & & \\
\hline & & & $53-2,50-54$ & & $16 \cdot 6,30-32$ \\
\hline & & $29 \mathrm{Tm} \mathrm{D.} \mathrm{hughesi}$ & $\begin{array}{c}54-1,50-54 \\
50\end{array}$ & & $\begin{array}{c}17-1,30-32 \\
75\end{array}$ \\
\hline & & & $52-3,50-54$ & & $17-6,30-32$ \\
\hline & & $30 \mathrm{Tm} D$. ontongensis & $\begin{array}{c}54-1,50-54 \\
75\end{array}$ & & $\begin{array}{c}18-2,90-92 \\
50\end{array}$ \\
\hline
\end{tabular}




\section{DETAILS OF MORPHOLOGICAL CHANGE IN DIDYMOCYRTIS}

Because they are less likely to be distorted or contaminated, sequences sampled by the hydraulic piston corer will provide more detailed and reliable information on the course of evolutionary changes than do sequences obtained in soft sediments by rotary drilling. We made a preliminary exploration of this potential by examining the change in the size of the polar caps in the genus Didymocyrtis, through Hole 503A. It has long been known (Riedel, 1957, 1959; Riedel and Sanfilippo, 1970) that the polar caps in this lineage increase in size through the late Cenozoic, but there has been little opportunity to determine whether the rate of increase has been uniform. Westberg and Riedel (1978) attempted to evaluate this in several sequences rotary-drilled in the tropical Pacific, but the results were undoubtedly influenced by distortion of columns sampled in this way (Gealy et al., 1971; Moore, 1972b). Kellogg (1980) investigated the tendency through the middle and lower Miocene in a piston core in which the sequence was believed to be undistorted, but she measured aspects of the morphology not directly comparable with those investigated by other authors.

We measured the height of the polar caps and length of cortical shell in 20-40 specimens of Didymocyrtis from 27 samples from Hole 503A. The measurements and calculations were facilitated by the microprocessorbased device described by Budai et al. (1980). Original samples were chosen for being relatively free of reworking and approximately evenly spaced along the column, and intermediate samples were added later at levels bracketing abrupt morphological changes. Results are summarized in Table 4 and plotted in Figure 3, A and B. Figure $3 \mathrm{~A}$ shows that the tendency for the relative size

Table 4. Measurements (in $\mu \mathrm{m}$ ) on polar caps and cortical shells of Didymocyrtis spp. in Hole 503A. The last group of columns refer to the ratio of polar cap height to cortical shell length.

\begin{tabular}{|c|c|c|c|c|c|c|c|c|}
\hline \multirow{2}{*}{$\begin{array}{l}\text { Core/Section } \\
\text { (interval in } \mathrm{cm} \text { ) }\end{array}$} & \multirow[b]{2}{*}{$\mathrm{N}$} & \multicolumn{2}{|c|}{ Polar Cap } & \multicolumn{2}{|c|}{ Cortical Shell } & \multicolumn{3}{|c|}{ Ratio } \\
\hline & & Mean & SD & Mean & SD & Mean & SD & Median \\
\hline $1-1,50-54$ & 40 & 47.3 & 7.06 & 108.1 & 11.55 & 0.44 & 0.06 & 0.44 \\
\hline $2-1,100-102$ & 27 & 46.4 & 6.15 & 110.6 & 9.21 & 0.42 & 0.06 & 0.43 \\
\hline $7-1,50-54$ & 12 & 33.7 & 5.82 & 109.5 & 10.40 & 0.31 & 0.06 & 0.30 \\
\hline $10-1,50-54$ & 17 & 32.6 & 4.94 & 106.1 & 12.50 & 0.31 & 0.04 & 0.30 \\
\hline $11-2,50-54$ & 16 & 33.9 & 4.96 & 111.4 & 9.87 & 0.31 & 0.05 & 0.31 \\
\hline $12-1,50-54$ & 33 & 33.4 & 5.06 & 105.3 & 9.09 & 0.32 & 0.05 & 0.31 \\
\hline $14-3,50-54$ & 31 & 34.2 & 4.25 & 107.9 & 9.30 & 0.32 & 0.04 & 0.31 \\
\hline $15-3,50-54$ & 40 & 36.9 & 4.95 & 114.9 & 9.07 & 0.32 & 0.04 & 0.32 \\
\hline $19-2,60-64$ & 40 & 33.3 & 4.20 & 101.5 & 11.28 & 0.33 & 0.05 & 0.34 \\
\hline $20-1,50-54$ & 39 & 35.2 & 4.48 & 126.4 & 17.00 & 0.28 & 0.04 & 0.27 \\
\hline $20-2,50-54$ & 23 & 32.4 & 4.06 & 120.2 & 15.60 & 0.28 & 0.05 & 0.26 \\
\hline $20-3,50-54$ & 40 & 33.6 & 4.92 & 103.5 & 12.30 & 0.33 & 0.06 & 0.34 \\
\hline $21-1,50-54$ & 27 & 33.7 & 6.13 & 117.2 & 15.61 & 0.29 & 0.07 & 0.27 \\
\hline $21-2,50-54$ & 40 & 34.2 & 5.02 & 111.6 & 14.65 & 0.31 & 0.06 & 0.30 \\
\hline $24-3,50-54$ & 40 & 35.6 & 4.28 & 101.9 & 12.40 & 0.35 & 0.05 & 0.35 \\
\hline $30-1,50-54$ & 40 & 36.0 & 5.26 & 107.4 & 11.58 & 0.34 & 0.05 & 0.34 \\
\hline $36-1,51-54$ & 40 & 33.4 & 4.98 & 108.1 & 12.37 & 0.31 & 0.04 & 0.31 \\
\hline $41-1,50-54$ & 40 & 33.4 & 3.88 & 112.6 & 11.44 & 0.30 & 0.04 & 0.30 \\
\hline $42-1,50-54$ & 46 & 31.1 & 4.30 & 113.5 & 10.04 & 0.28 & 0.04 & 0.28 \\
\hline $43-1,50-54$ & 40 & 31.1 & 4.48 & 123.4 & 13.60 & 0.25 & 0.04 & 0.25 \\
\hline $44-2,50-54$ & 40 & 32.1 & 5.63 & 130.5 & 8.83 & 0.25 & 0.04 & 0.24 \\
\hline $44-3,50-54$ & 40 & 30.4 & 5.42 & 126.7 & 9.21 & 0.24 & 0.04 & 0.24 \\
\hline $48-2,50-54$ & 40 & 32.5 & 4.07 & 110.3 & 5.87 & 0.30 & 0.04 & 0.29 \\
\hline $50-2,50-54$ & 40 & 33.4 & 2.95 & 108.6 & 6.90 & 0.31 & 0.04 & 0.31 \\
\hline $54-1,50-54$ & 40 & 30.6 & 3.81 & 104.9 & 7.42 & 0.29 & 0.04 & 0.29 \\
\hline
\end{tabular}

of the caps to increase during most of the upper Miocene and Pliocene is slight, if it exists at all, though above and below this interval the increase through time is pronounced. Interrupting the central plateau of the graph are two intervals showing the proportion of cap height to cortical shell length to be depressed. That this is not due to an interval of comparatively rapid sedimentation is evident from the rate of accumulation curve for this hole (see site chapter, this volume). When cap height and cortical shell length are considered separately (Fig. 3B), they are seen to vary sympathetically above the middle of Core 503A-20 and antipathetically below it. The proportion plotted in Figure $3 \mathrm{~A}$ is seen to be controlled mainly by the height of the caps.

It would seem promising to investigate this change at other locations, to determine the geographic extent of the morphological changes expressed here. In that way it may be possible to determine the extent to which the changes are either genetic or phenotypic reactions to environmental differences.

\section{SPECIES LIST}

The purpose of this list is to provide bibliographic references to the taxa mentioned in this chapter. The only literature references given are to the original description and to our present concept of the species if it differs from or is more detailed than the original.

Acrobotrys tritubus Riedel

Acrobotrys tritubus Riedel, 1957, p. 80, pl. 1, fig. 5.

Amphirhopalum ypsilon Haeckel

Amphirhopalum ypsilon Haeckel, 1887, p. 522; Nigrini, 1971, p. 447 , pl. 34.1, figs. $7 \mathrm{a}-\mathrm{c}$.

Anthocyrtidium angulare Nigrini

Anthocyrtidium angulare Nigrini, 1971 , p. 445 , pl. 34.1 , figs. $3 \mathrm{a}, \mathrm{b}$.

Axoprunum angelinum (Campbell and Clark)

Stylosphaera angelina Campbell and Clark, 1944, p. 12, pl. 1, figs. 14-20.

Stylatractus universus Hays, 1970, p. 215, pl. 1, figs. 1, 2.

Axoprunum angelinum (Campbell and Clark)-Kling, 1973, p. 634 , pl. 1, figs. 13-16, pl. 6, figs. 14-18.

Buccinosphaera invaginata $\mathrm{Haeckel}$

Buccinosphaera invaginata Haeckel, 1887, p. 99, pl. 5, fig. 11; Strelkov and Reshetyak, 1962, p. 129 and 137, fig. 12; Nigrini, 1971 , p. 445, pl. 34.1, fig. 2; Knoll and Johnson, 1975, p. 63, pl. 1, figs. 3-6.

Remarks: We continue to use the rather unsatisfactory generic classification of collosphaerids proposed by Strelkov and Reshetnyak (1971) rather than the modified one suggested by Bjørklund and Goll (1979), because the latter is almost as unsatisfactory in that it does not admit the possibility of eventual recognition of Ehrenberg's species (which would, for example, make Tetrasolenia Ehrenberg 1860 the objective senior synonym of Solenosphaera, as a result of Chediya's 1959 designation of a junior synonym of Tetrasolenia quadrata as the type species of that genus) (Foreman and Riedel, 1972).

Calocycletta robusta Moore group

Calocycletta robusta Moore, 1971, p. 743, pl. 10, figs. 5, 6. Calocycletta caepa Moore, 1972a, p. 150, pl. 2, figs. 4-7.

Calocycletta robusta Moore group-Riedel and Sanfilippo, 1978a, p. 66 , pl. 3 , figs. 10,11 .

Centrobotrys thermophila Petrushevskaya Centrobotrys thermophila Petrushevskaya, 1965, p. 115, fig. 20.

Collosphaera tuberosa Haeckel

Collosphaera tuberosa Haeckel, 1887, p. 97; Nigrini, 1971, p. 445, pl. 34.1, fig. 1; Strelkov and Reshetnyak, 1971, pp. 336-337, pl. 4, figs. 24, 25; Knoll and Johnson, 1975, p. 63, pl. 2, figs. 1-3.

Diartus hughesi (Campbell and Clark)

Ommatocampe hughesi Campbell and Clark, 1944, p. 23, pl. 3, fig. 12. 


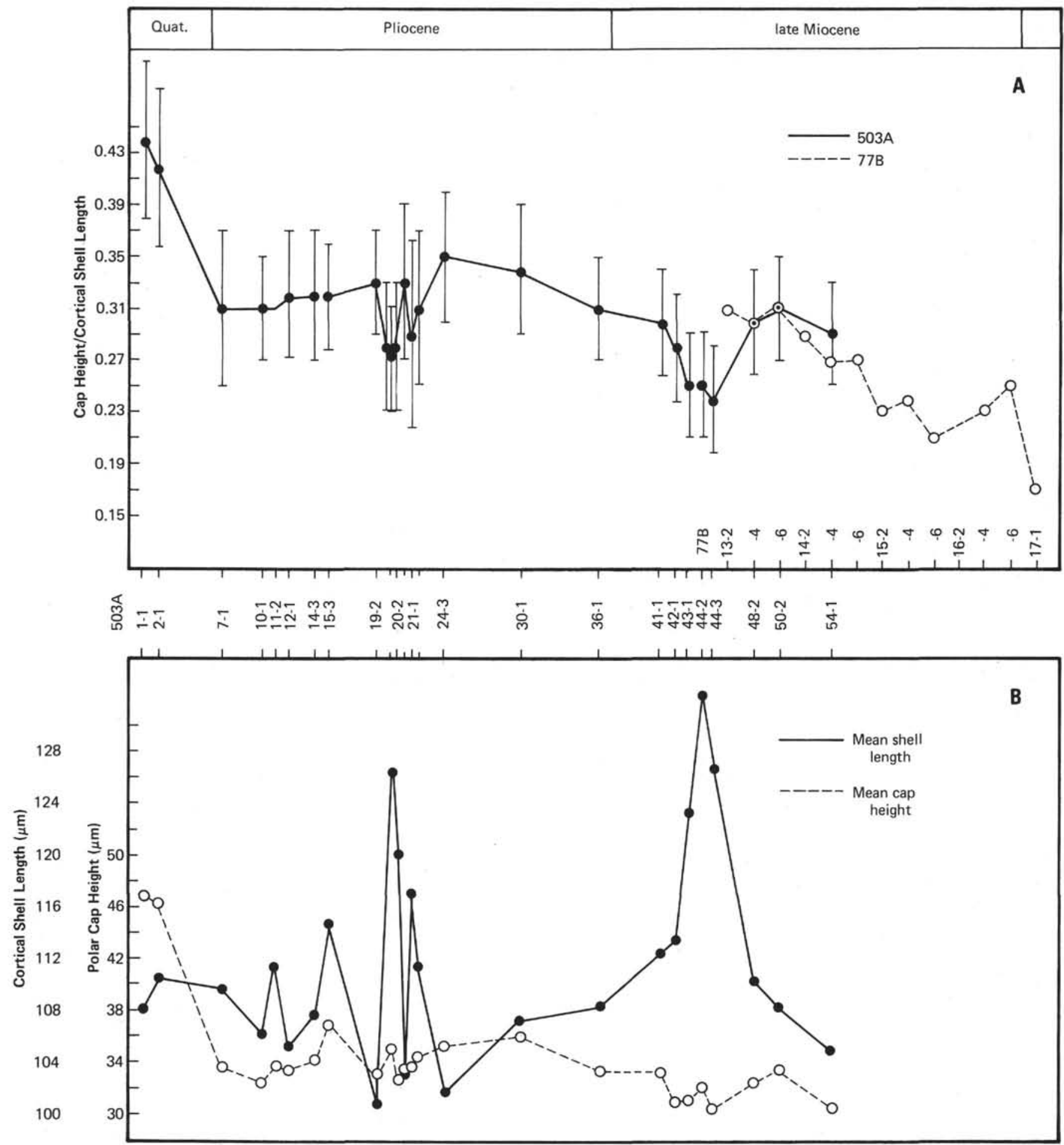

Figure 3. A. Ratio of polar cap height to cortical shell length in Didymocyrtis spp. from Holes 503A and 77B (from Westberg and Riedel, 1978, p. 13). Points represent the mean of 20-40 measurements; bars represent standard deviation. B. Polar caps height and cortical shell length of Didymocyrtis spp. in samples from Hole 503A.

Ommatartus hughesi (Campbell and Clark) -Riedel and Sanfilippo, 1978a, p. 71, pl. 7, fig. 7.

Diartus hughesi (Campbell and Clark)-Sanfilippo and Riedel, 1980 , p. 1010 , pl. 1, fig. i.

Diartus petterssoni (Riedel and Sanfilippo)

Cannartus (?) petterssoni Riedel and Sanfilippo, 1970, p. 520, pl. 14 , fig. $3 ; 1978$ a, p. 67 , pl. 4 , fig. 2 .
Diartus petterssoni (Riedel and Sanfilippo)-Sanfilippo and Riedel, 1980 , p. 1010 , pl. 1, fig. h.

Dictyocoryne ontongensis Riedel and Sanfilippo

Dictyocoryne ontongensis Riedel and Sanfilippo, 1971, p. 1588 , pl. 1E, figs. 1, 2, pl. 4, figs. 9-11; 1978a, p. 68, pl. 5, fig. 1 . Didymocyrtis antepenultima (Riedel and Sanfilippo)

Ommatartus antepenultimus Riedel and Sanfilippo, 1970, p. 521, 
pl. 14, fig. 4; Westberg and Riedel, 1978, p. 22, pl. 2, figs. 4, 5. Didymocyrtis antepenultima (Riedel and Sanfilippo)-Sanfilippo and Riedel, 1980, p. 1010.

Didymocyrtis avita (Riedel)

Panartus avitus Riedel, 1953 , p. 808 , pl. 84 , fig. 7.

Didymocyrtis avita (Riedel)-Sanfilippo and Riedel, 1980, p. 1010.

Didymocyrtis penultima (Riedel)

Panarium penultimum Riedel, 1957, p. 76, pl. 1, fig. 1

Ommatartus penultimus (Riedel)-Westberg and Riedel, 1978, p. 22 , pl. 2, figs. 6-8.

Didymocyrtis penultima (Riedel)-Sanfilippo and Riedel, 1980, p. 1010 , pl. 1, fig. f.

Didymocyrtis tetrathalamus (Haeckel)

Panartus tetrathalamus Haeckel, 1887, p. 378, pl. 40, fig. 3.

Didymocyrtis tetrathalamus (Haeckel)-Sanfilippo and Riedel, 1980 , p. 1010, pl. 1, fig. g.

Lamprocyrtis heteroporos (Hays)

Lamprocyclas heteroporos Hays, 1965, p. 179, pl. 3, fig. 1.

Lamprocyrtis heteroporos (Hays)-Kling, 1973, p. 639, pl. 5, figs.

19-21, pl. 15, fig. 6.

Lamprocyrtis neoheteroporos $\mathrm{Kling}$

Lamprocyrtis neoheteroporos Kling, 1973, p. 639, pl. 5, figs. 17, 18 , pl. 15 , figs. 4,5 .

Lamprocyrtis nigriniae (Caulet)

Conarachnium nigriniae Caulet, 1971, p. 3, pl. 3, figs. 1-4, pl. 4, figs. 1-4.

Lamprocyrtis haysi Kling, 1973, p. 639, pl. 5, figs. 15, 16, pl. 15, figs.1-3.

Lamprocyrtis nigriniae (Caulet)-Kling, 1977, p. 217, pl. 1, fig. 17.

Lithopera bacca Ehrenberg

Lithopera bacca Ehrenberg, 1872, p. 314; Riedel and Sanfilippo, 1978 a, p. 70 , pl. 6, fig. 9 .

Lychnodictyum audax Riedel

Lychnodictyum audax Riedel, 1953, p. 810, pl. 85, fig. 9.

Phormostichoartus doliolum (Riedel and Sanfilippo)

Artostrobium doliolum Riedel and Sanfilippo, 1971, p. 1599, pl. $1 \mathrm{H}$, figs. 1-3, pl. 8, figs. 14, 15; Westberg and Riedel, 1978, p. 20, pl. 3, figs. 10, 11 .

Phormostichoartus doliolum (Riedel and Sanfilippo)-Nigrini, 1977, pp. 252, 253, pl. 1, fig. 14.

Phormostichoartus fistula Nigrini

Phormostichoartus fistula Nigrini, 1977, p. 253, pl. 1, figs. 11-13.

Pterocanium prismatium Riedel

Pterocanium prismatium Riedel, 1957, p. 87, pl. 3, figs. 4, 5; Riedel and Sanfilippo, 1978a, p. 72, pl. 9, fig. 1.

Pterocorys hertwigii (Haeckel)

Eucyrtidium hertwigii Haeckel, 1887, p. 1491, pl. 80, fig. 12.

Pterocorys hertwigii (Haeckel)-Petrushevskaya, 1972, fig. 1(10).

Siphostichoartus corona (Haeckel)

Cyrtophormis (Acanthocyrtis) corona Haeckel, 1887, p. 1462, pl.

77, fig. 15.

Phormostichoartus corona (Haeckel)-Riedel and Sanfilippo, 1978a, p. 71, pl. 7, fig. 12.

Siphostichoartus corona (Haeckel)-Nigrini, 1977, p. 257, pl. 2, figs. 5-7.

Solenosphaera omnitubus Riedel and Sanfilippo

Solenosphaera omnitubus Riedel and Sanfilippo, 1971, p. 1586, pl. 1A, fig. 24, pl. 4, figs. 1, 2; 1978a, p. 73, pl. 9, figs. 8, 9.

Spirocyrtis gyroscalaris Nigrini

Spirocyrtis gyroscalaris Nigrini, 1977, p. 258, pl. 2, figs. 10, 11.

Spirocyrtis scalaris Haeckel

Spirocyrtis scalaris Haeckel, 1887, p. 1509, pl. 76, fig. 14; Nigrini, 1977 , p. 259 , pl. 2, figs. $12,13$.

Spongaster berminghami (Campbell and Clark)

Spongasteriscus berminghami Campbell and Clark, 1944, p. 30, pl. 5, figs. 1, 2.

Spongaster berminghami (Campbell and Clark)-Riedel and Sanfilippo, 1978a, p. 73, pl. 2, figs. 14-16.

Spongaster pentas Riedel and Sanfilippo

Spongaster pentas Riedel and Sanfilippo, 1970, p. 523, pl. 15, fig. 3; 1978a, p. 74, pl. 2, figs. 5-8.

Spongaster tetras Ehrenberg

Spongaster tetras Ehrenberg, 1860, p. 833; Riedel and Sanfilippo, 1978a, p. 74, pl. 2, figs. 2, 3.
Spongodiscus ambus Sanfilippo and Riedel

Spongodiscus ambus Sanfilippo and Riedel, 1974, p. 1024, pl. 1, figs. 12-14.

Stichocorys delmontensis (Campbell and Clark)

Eucyrtidium delmontense Campbell and Clark, 1944, p. 56, pl. 7, figs. 19, 20.

Stichocorys delmontensis (Campbell and Clark)-Westberg and Riedel, 1978, p. 22, pl. 3, figs. 1-5.

Stichocorys peregrina (Riedel)

Eucyrtidium elongatum peregrinum Riedel, 1953, p. 812, pl. 85, fig. 2 .

Stichocorys peregrina (Riedel)-Westberg and Riedel, 1978, p. 22, pl. 3, figs. 6-9.

Stichocorys wolffii Haeckel

Stichocorys wolffii Haeckel, 1887, p. 1479, pl. 80, fig. 10; Riedel and Sanfilippo, 1978a, p. 74, pl. 9, fig. 12.

Theocorythium trachelium (Ehrenberg)

Eucyrtidium trachelius Ehrenberg, 1872, p. 312.

Theocorythium trachelium (Ehrenberg)-Nigrini, 1967, p. 77, pl.

8, figs. 1, 2, pl. 9, figs. 1, 2; Riedel and Sanfilippo, 1978a, p. 76, pl. 9, fig. 17 .

Theocorythium vetulum Nigrini

Theocorythium vetulum Nigrini, 1971, p. 447, pl. 34.1, figs. 6a, b.

\section{ACKNOWLEDGMENTS}

This study was supported by the National Science Foundation Grant No. OCE79-20265. We are grateful to S. A. Kling and Annika Sanfilippo for reviewing the manuscript. M. A. Hanger competently typed the manuscript and assisted with the proofreading. Evelyn Portillo drafted the text-figures.

\section{REFERENCES}

Bjørklund, K. R., and Goll, R. M., 1979. Internal skeletal structures of Collosphaera and Trisolenia: a case of repetitive evolution in the Collosphaeridae (Radiolaria). J. Paleontol., 53(No. 6): 1293-1326.

Budai, Alexis, Riedel, W. R., and Westberg, M. J., 1980. A general purpose paleontologic information device. J. Paleontol., 54(No. 1): $259-262$.

Campbell, A. S., and Clark, B. L., 1944. Miocene radiolarian faunas from southern California. Geol. Soc. Am. Spec. Pap., 51:1-76.

Caulet, J. P., 1971. Contribution a l'etude de quelques radiolaires nassellaires des boues de la Méditerranée et du Pacifique. Cah. micropaléontol., 2(№. 10):1-10.

Chediya, D. M., 1959. Obzor Sistematiki Radiolyarii: Stalinabad (Tadzhikskii Gosudarstvennyi Universitet).

Ehrenberg, C. G., 1860. Über den Tiefgrund des stillen Oceans zwischen Californien und den Sandwich-Inseln aus bis 15600' Tiefe nach Lieut. Brooke. K. Preuss. Akad. Wiss. Monatsb., pp. 819-833.

1872. Mikrogeologischen Studien als Zusammenfassung seiner Beobachtungen des kleinsten Lebens der Meeres-Tiefgründe aller Zonen und dessen geologischen Einfluss. K. Preuss. Akad. Wiss. Monatsb., pp. 265-322.

1873. Grossere Felsproben des Polycystinen-Mergels von Barbados mit weiteren Erläuterungen. K. Preuss. Akad. Wiss. Monatsb., pp. 213-263.

Foreman, H. P., 1973. Radiolaria of Leg 10 with systematics and ranges for the families Amphipyndacidae, Artostrobiidae, and Theoperidae. In Worzel, J. L., Bryant, W., et al., Init. Repts. DSDP, 10: Washington (U.S. Govt. Printing Office), 407-474.

Foreman, H. P., and Riedel, W. R., 1972. Catalogue of Polycystine Radiolaria (Ser. 1, Vol. 1, Pt. 2): New York (Micropaleontology Press).

Gealy, E. L., Winterer, E. L., and Moberly, R. M., Jr., 1971. Methods, Conventions and General Observations. In Winterer, E. L., Riedel, W. R., et al., Init. Repts. DSDP, 7, Pt. 2: Washington (U.S. Govt. Printing Office), 9-26.

Haeckel, E., 1887. Report on the Radiolaria collected by H.M.S. Challenger during the years 1873-76. Report on the Scientific Results of the Voyage of the H.M.S. Challenger, Zoology, 18:1-1303.

Harting, P., 1863. Bijdrage tot de kennis der mikroskopische faune en flora van de Banda-Zee, naar aanleiding van een onderzoek van eenige door diepzeeloodingen van 990 tot 4000 vademen uit die zee opgebragte gronden. K. Akad. Wet., 10:1-34. 
Hays, J. D., 1965. Radiolaria and late Tertiary and Quaternary history of Antarctic seas: Biology of the Antarctic Seas. II: Antarct. Res. Ser., 5:125-184. 1970. Stratigraphy and evolutionary trends of radiolaria in North Pacific deep sea sediments. In Hays, J. D. (Ed.), Geological Investigations of the North Pacific: Geological Society of America Memoir 126, 185-218.

Kellogg, D. E., 1980. Character displacement and phyletic change in the evolution of the radiolarian subfamily Artiscinae. Micropaleontology, 26(No. 2):196-210.

Kling, S. A., 1973. Radiolaria from the eastern North Pacific, Deep Sea Drilling Project, Leg 18. In Kulm, L. D., von Huene, R., et al., Init. Repts. DSDP, 18: Washington (U.S. Govt. Printing Office), 617-672.

1977. Local and regional imprints on radiolarian assemblages from California coastal basin sediments. Mar. Micropaleont., 2(No. 3):207-221.

Knoll, A. H., and Johnson, D. A., 1975. Late Pleistocene evolution of the collosphaerid radiolarian Buccinosphaera invaginata Haeckel. Micropaleontology, 21(No. 1):60-68.

Moore, T. C., Jr., 1971. Radiolaria. In Tracey, J. I., Jr., Sutton, G. H., et al., Init. Repts. DSDP, 8: Washington (U.S. Govt. Printing Office), $727-775$.

1972a. Mid-Tertiary evolution of the radiolarian genus $\mathrm{Cal}$ ocycletta. Micropaleontology, 18:144-152.

1972b. DSDP successes, failures, proposals. Geotimes, 7 (No. 7):27-31.

Nigrini, C., 1967. Radiolaria in pelagic sediments from the Indian and Atlantic Ocean. Bull. Scripps Inst. Oceanog., 11:1-125. 1971. Radiolarian zones in the Quaternary of the equatorial Pacific Ocean. In Funnell, B. M., and Riedel, W. R. (Eds.), The Micropalaeontology of the Oceans: Cambridge (Cambridge University Press), pp. 443-461.

, 1977. Tropical Cenozoic Artostrobiidae (Radiolaria). Micropaleontology, 23(No. 3):241-269.

Petrushevskaya, M. G., 1965. Osobennosti konstryktsii skeleta radiolyarii Botryoidae (otr. Nassellaria). Tr. Zool. Inst., Akad. Nauk. SSSR., 35:79-118.

1972. Biostratigrafiya glubokovodnykh chetvertichnykh osadkov po dannym radiolyarievogo analyza. Okeanologiya, 12 (No. 1):71-86.

Riedel, W. R., 1953. Mesozoic and late Tertiary Radiolaria of Rotti. J. Paleontol., 27:805-813.

1957. Radiolaria: a preliminary stratigraphy. Rep. Swed. Deep-Sea Exped., 6:59-96.

1959. Oligocene and Lower Miocene Radiolaria in tropical Pacific sediments. Micropaleontology, 5:285-302.

Riedel, W. R., and Sanfilippo, A., 1970. Radiolaria, Leg 4, Deep Sea Drilling Project. In Bader, R. G., Gerard, R. D., et al., Init.
Repts. DSDP, 4: Washington (U.S. Govt. Printing Office), 503-575.

1971. Cenozoic Radiolaria from the western tropical Pacific, Leg 7. In Winterer, E. L., Riedel, W. R., et al., Init. Repts. DSDP, 7, Pt. 2: Washington (U.S. Govt. Printing Office), 1529-1672.

1973. Cenozoic Radiolaria from the Caribbean, Deep Sea Drilling Project, Leg 15. In Edgar, N. T., Saunders, J. B., et al., Init. Repts. DSDP, 15: Washington (U.S. Govt. Printing Office), 705-751.

, 1978a. Stratigraphy and evolution of tropical Cenozoic radiolarians. Micropaleontology, 24(No. 1):61-96. $81-128$.

Sanfilippo, A., and Riedel, W. R., 1973. Cenozoic Radiolaria (Exclusive of theoperids, artostrobiids and amphipyndacids) from the Gulf of Mexico, DSDP Leg 10. In Worzel, J. L., Bryant, W., et al., Init. Repts. DSDP, 10: Washington (U.S. Govt. Printing Office), 475-611.

1974. Radiolaria from the West-Central Indian Ocean and Gulf of Aden, DSDP Leg 24. In Fisher, R. L., Bunce, E. T., et al., Init. Repts. DSDP, 24: Washington (U.S. Govt. Printing Office), 997-1035.

1975. Late Tertiary Radiolarians from Crete. VI Congress Regional Committee on Mediterranean Neogene Stratigraphy, Bratislava, 1975. pp. 61-74.

1976. Radiolarian occurrences in the Caribbean Region. Publication de la VII conférence géologique des Caraibes du 30 juin au 12 juillet 1974 (VII Conférence Géologique des Caraibes, Cayenne, Département Francais de la Guyane), pp. 145-168.

1980. A revised generic and suprageneric classification of the Articins (Radiolaria). J. Paleontol., 54(No. 5):1008-1012.

Strelkov, A. A., and Reshetnyak, V. V., 1962. Kolonialnye radiolyarii Spumellaria yuzhno-kitaiskogo morya (raion yuzhoy okonechnosti ostrova Hainan). Studia Mar. Sin., 1:121-139.

,1971. Colonial Spumellarian radiolarians of the world ocean. In Strelkov, A. A. (Ed.), Exploration of the fauna of the Seas: Moscow (Zoological Institute, Academy of Sciences, U.S.S.R.), IX (XVII):295-373.

Theyer, F., Mato, C. Y., and Hammond, S. R., 1978. Paleomagnetic and geochronologic calibration of latest Oligocene to Pliocene radiolarian events, equatorial Pacific. Mar. Micropaleontol., 3: 377-395.

Westberg, M. J., and Riedel, W. R., 1978. Accuracy of radiolarian correlations in the Pacific Miocene. Micropaleontology, 24(No. 1):1-23.

, in press. Radiolarians from the Mid-America trench off Guatemala, DSDP Leg 67. In von Huene, R., Aubouin, J., et al., Init. Repts. DSDP, 67: Washington (U.S. Govt. Printing Office). 